\title{
Europas politische Verfasstheit im Lichte des Fiskalvertrages
}

von Frank Schorkopf

Der seit Dezember 2011 mit beträchtlicher Geschwindigkeit formulierte und beschlossene ,,Vertrag über Stabilität, Koordinierung und Steuerung in der Wirtschafts- und Währungsunion", kurz Fiskalvertrag, stellt Wissenschaft und Praxis vor maßgebliche Herausforderungen. Der vorliegende Beitrag summiert die Substanz des Fiskalvertrages und skizziert den Zusammenhang zwischen der Normativität der europäischen Rechtsgemeinschaft und dem Ausweichen einer Mehrheit von Mitgliedstaaten auf die intergouvernementale Ebene, um auf dieser Grundlage die Folgen für die Idee der europäischen Integration zu analysieren. Abschließend wird dies um eine Diskussion der Auswirkungen auf das deutsche Verfassungsgefüge ergänzt.

The "Treaty on Stability, Coordination and Governance in the Economic and Monetary Union", written and decided at a remarkably fast pace and better known as the Fiscal Compact, constitutes a major challenge both to the political process and the related academic disciplines. This contribution summarises the substance of the Treaty and outlines the link between the normativity of European law and the evasion of a majority of Member States to the intergovernmental level. Subsequently, the discussion turns to the consequences of these developments for the idea of European integration and traces their effects on the German constitutional framework.

\section{Kontext der Krise}

Wenn Historiker auf unsere Zeit zurückschauen und über die Europäische Union forschen werden, dann wird der ,Vertrag über Stabilität, Koordinierung und Steuerung in der Wirtschafts- und Währungsunion“ ein ertragreicher Studiengegenstand sein. Dieser Fiskalvertrag (FV), der seit Dezember 2011 für ein paar Wochen in amtlicher Sprache dunkel-assoziativ auch als Fiskalpakt benannt war, ${ }^{1}$ steht mit Inhalt, Form und Kontext typenhaft für die europäische Integrati-

1 In der Erklärung der Staats- und Regierungschefs des Eurowährungsgebiets v. 9.12.2011 wird die Bezeichnung fiscal compact verwendet, die in der deutschen Fassung der Erklärung mit „fiskalpolitischer Pakt“ übersetzt wird. In neueren Dokumenten wird der Vertrag nicht mehr als „Pakt“, sondern als „Fiskalvertrag“ bezeichnet; ein Grund für diesen Namenswechsel könnte sein, dass Titel III FV seit dem zweiten Entwurf überschrieben ist mit „Fiskalpolitischer Pakt“. Der Vertragstext nebst Protokoll ist in 
on der Gegenwart. Mit dem Vertrag wollen die Mitgliedstaaten in der Staatsschuldenkrise das Euro-Währungsgebiet kräftigen, ihre Haushaltsdisziplin fördern und die nationalen Wirtschaftspolitiken besser aufeinander abstimmen. Sie gehen diesen Schritt aber ohne den Konsens aller Mitgliedstaaten, weichen deshalb auf den klassisch völkerrechtlichen Vertrag außerhalb des EU-Rechts aus und stellen eine für Europas politische Verfasstheit womöglich entscheidende Weiche.

Der Fiskalvertrag entstand innerhalb weniger Wochen. Diese begannen mit dem Treffen des deutsch-französischen „Direktorats“"2 am 5. Dezember und mit der politischen Einigung der Staats- und Regierungschefs der Euro-Staaten am 9. Dezember 2011, ${ }^{3}$ erreichten am 31. Januar 2012 den Verhandlungsabschluss und endeten mit der Unterzeichnung durch die Staats- und Regierungschefs von 25 Mitgliedstaaten am 2. März 2012. ${ }^{4}$ Seine Vorgeschichte reicht bis zu der Entscheidung für die Wirtschafts- und Währungsunion im Unionsvertrag von Maastricht zurück. Sie ist jedoch unmittelbar verbunden mit den Ereignissen auf den Finanzmärkten seit Mitte des Jahres 2007, die sich zu einer europäischen Staatsschuldenkrise und inzwischen zu einer Gesamthandskrise der Europäischen Union und ihrer Mitgliedstaaten erweitert haben. ${ }^{5}$

Das nähere Verständnis des Fiskalvertrages erschließt sich in hervorragender Weise über ein Dokument aus dem Auswärtigen Amt. Es handelt von den integrationspolitischen Fortschritten, die erforderlich seien, um eine Stabilitätsunion zu schaffen. Den Mitgliedern des Deutschen Bundestages wurde es vom Außenminister mit Brief vom 20. Oktober 2011 übermittelt. ${ }^{6}$ Nach diesem Strate-

einer deutsch-englischen Fassung zugänglich im Entwurf der Bundesregierung für ein Zustimmungsgesetz: BR-Drs. 130/12, 7-19 = BT-Drs. 17/9046, 6-17.

2 Statements von Bundeskanzlerin Angela Merkel und dem französischen Staatspräsidenten Nicolas Sarkozy v. 5.12.2011; einzelne Elemente, wie etwa die Schuldenbremse, wurden bereits in einem Gemeinsamen Deutsch-Französischen Brief an EU-Ratspräsident Herman Van Rompuy erwähnt, August 2011. Erhellend zum deutsch-französischen Handeln vgl. Rinke, A.: Europäische Sternstunde. Wie „Merkozy“ die Grundlagen eines neuen Europas schufen, in: Internationale Politik 67 (2012), 8-20.

3 Europäischer Rat: Erklärung der Staats- und Regierungschefs des Eurowährungsgebiets v. 9.12.2011.

4 Europäischer Rat: Schlussfolgerungen der Tagung v. 1./2.3.2012, EUCO 4/12, 2. Der Vertrag soll am 1.1.2013 in Kraft treten, sofern zwölf Euro-Staaten diesen bis dahin ratifiziert haben werden, Art. 14 Abs. 2 FV. Nach dem Willen der Bundesregierung soll der Bundestag Ende März 2012 in die erste Lesung eintreten und dann bis Ende Mai beraten. Mitte Juni soll der Bundesrat zustimmen.

5 Einen Überblick der Ereignisse und institutionellen Reaktionen geben Kunstein, T./Wessels, W.: Die Europäische Union in der Währungskrise: Eckdaten und Schlüsselentscheidungen, in: integration 34 (2011), 308-322.

6 Auswärtiges Amt: Schreiben des Bundesministers des Auswärtigen v. 20.10.2011 nebst Anlage; verteilt als Ausschuss-Drucksache u.a. des Europaausschusses im Deutschen Bundestag (A-Drs. 17(21)778). 
giepapier müsse sich Deutschland vier Herausforderungen stellen, um „die gegenwärtige Staatsschuldenkrise im Euro-Raum dauerhaft zu überwinden“. Neben kurzfristigem Krisen-Management seien entscheidende Schritte hin zu einer „echten Stabilitätsunion“ zu unternehmen, d.h. es müsse „eine Kultur solider Haushaltsführung verbindlich und fest" verankert werden. Bei der Errichtung einer solchen wirtschafts- und finanzpolitischen Stabilitätsunion stellten sich „Fragen der politischen Verfasstheit Europas in neuem Licht“; Europa müsse die Finanzverfassung gegeben werden, die es brauche.

Der Fiskalvertrag verkörpert einerseits das erfolgreiche Ergebnis dieser politischen Strategie und steht andererseits für deren vorläufiges Scheitern. Das Scheitern ist darin zu sehen, dass es nicht gelungen ist, die konkreten Schritte hin zu einer Stabilitätsunion im institutionellen Rahmen des Primärrechts zu gehen. ${ }^{7}$ Großbritannien und auch die Tschechische Republik haben sich entschieden, eine entsprechende Änderung des AEU-Vertrages nicht mitzutragen. ${ }^{8}$ Es blieb deshalb nur der Weg über eine Vereinbarung auf intergouvernementaler Ebene, d.h. der Abschluss eines völkerrechtlichen Vertrages zwischen den beteiligungswilligen Mitgliedstaaten. Der Erfolg besteht darin, dass es der deutschen Europapolitik weitgehend gelungen ist, ihr materielles Konzept in Rechtsnormen zu übersetzen und damit einen bislang ad hoc gestalteten Prozess wieder zu verrechtlichen. Der bestehende Stabilitäts- und Wachstumspakt wird ergänzt werden durch eine völkerrechtliche Verpflichtung zur Einhaltung einer strikteren Defizitgrenze, automatische Sanktionen bei Defizitverstößen, einen Rechtsweg zum Europäischen Gerichtshof und durch Einführung von Schuldenbremsen im nationalen Recht.

Es ist für das Verständnis des Fiskalvertrages außerdem wichtig, dass zu dem Zeitpunkt, an dem der Vertrag abgeschlossen wurde, bereits zahlreiche Maßnahmen der Krisenreaktion in Kraft getreten oder zumindest vereinbart waren: So soll das wirtschaftspolitische Zusammenwirken der Euro-Staaten durch eine „Arbeitsgruppe Wirtschaftspolitische Steuerung ${ }^{\star 9}$ und den so genannten Euro-

7 Bundesfinanzminister Wolfgang Schäuble erwähnt ausdrücklich die Revision der vertraglichen Grundlagen, um die aktuellen Herausforderungen zu bewältigen, in: ZSE 9 (2011), 301-304.

8 Vgl. Europäischer Rat: Erklärung der Staats- und Regierungschefs des Eurowährungsgebiets vom 9.12.2011, 7; Großbritannien wird nicht ausdrücklich genannt, ist aber der einzige Mitgliedstaat, der bei der Aufzählung fehlt. Art. 48 Abs. 4 UAbs. 2 und Abs. 6 UAbs. 2 EUV können nach dem EU- und AEU-Vertrag nur einstimmig und nach Ratifikation durch die Mitgliedstaaten geändert werden.

9 Europäischer Rat: Schlussfolgerungen der Tagung v. 28./29.10.2010, EUCO 25/10, $1 \mathrm{f}$. 
Plus-Pakt vom März 2011 verbessert werden. ${ }^{10}$ Die währungspolitische Seite der Union soll dadurch gestärkt werden, dass eine neue Norm in den AEU-Vertrag eingefügt werden wird, ${ }^{11}$ die wiederum den Europäischen Stabilisierungsmechanismus (ESM) ermöglichen soll ${ }^{12}$ - beide Maßnahmen werden den befristeten Euro-Rettungsfonds ablösen und verstetigen. ${ }^{13}$ Der europäische Gesetzgeber hat schließlich im November 2011 sechs Sekundärrechtsakte angenommen, die den Stabilitäts- und Wachstumspakt substantiell sowohl im Hinblick auf die Verfahrensregeln als auch auf die materiellen Voraussetzungen ändern. ${ }^{14}$ Zwei weitere Sekundärrechtsakte hat die Kommission vorgeschlagen. ${ }^{15}$ Der Fiskalvertrag knüpft besonders an diese Sekundärrechtsakte an. Deutschland möchte deren

10 Der Euro-Plus-Pakt ist abgedruckt als Anlage I zu den Schlussfolgerungen der Staats- und Regierungschefs der Mitgliedstaaten des Euro-Währungsgebiets v. 11.3.2011, („Ein Pakt für den Euro“); s. auch Europäischer Rat: Schlussfolgerungen der Tagung v. 24./25.3.2011, EUCO 10/1/11 Rev 1, Anlage I.

11 Europäischer Rat: Schlussfolgerungen v. 16./17.12.2010, EUCO 30/10, Anlage I.; s. Unterrichtung durch die Bundesregierung: BR-Drs. 872/10, Deutscher Bundestag: BT-Drs. 17/4880 sowie den Beschluss v. 17.3.2011, Plenarprotokoll 17/96, 11015 C. Zu Kritik vgl. Häde, U.: Art. 136 AEUV - eine neue Generalklausel für die Wirtschafts- und Währungsunion?, in: Juristenzeitung 66 (2011), 333-340.

12 Europäischer Rat: Schlussfolgerungen des Vorsitzes v. 24./25.3.2011, EUCO 10/1/11 Rev 1, Anlage II; Bundesregierung: Entwurf eines Gesetzes zu dem Vertrag v. 2.2.2012 zur Einrichtung des Europäischen Stabilitätsmechanismus, BT-Drs. 17/9045, der Vertragstext (T/ESM/2012/DE) ist der Drucksache beigefügt. Zur Entwurfsfassung Kube, H./Reimer, E.: Die Sicherung der Europäischen Wirtschafts- und Währungsunion: Rückkehr in die Bahnen des Rechts, in: Zeitschrift für Gesetzgebung 26 (2011), 332-350.

13 Der Rettungsfonds, der wohlwollend auch als Rettungsschirm bezeichnet wird, besteht aus der Europäischen Finanzstabilisierungs-Fazilität, EFSF-Rahmenvertrag v. 7.6.2010, und der Verordnung (EU) Nr. 407/2010 des Rates v. 11.5.2011 zur Einführung eines europäischen Finanzstabilisierungsmechanismus (ESFM), ABl. EU 2011 Nr. L 118/1, für die Einzelheiten s. Nettesheim, M.: Der Umbau der europäischen Währungsunion, in: Kadelbach (Hg.): Nach der Finanzkrise, Baden-Baden, 2012, 31-77.

14 Das so genannte six-pack besteht aus insgesamt sechs Sekundärrechtsakten, die am 13.12.2011 in Kraft getreten sind. Es handelt sich dabei um vier Verordnungen des Europäischen Parlaments und des Rates v. 16.11.2011: VO (EU) Nr. 1173/2011 über die wirksame Durchsetzung der haushaltspolitischen Überwachung im Euro-Währungsgebiet; VO (EU) Nr. 1174/2011 über Durchsetzungsmaßnahmen zur Korrektur übermäßiger makroökonomischer Ungleichgewichte im Euro-Währungsgebiet; VO (EU) Nr. 1175/2011 zur Änderung der VO (EG) Nr. 1466/97 des Rates über den Ausbau der haushaltspolitischen Überwachung und der Überwachung und Koordinierung der Wirtschaftspolitiken und VO (EU) Nr. 1176/2011 über die Vermeidung und Korrektur makroökonomischer Ungleichgewichte; s. insgesamt AB1. EU 2011 Nr. L 306/1-32; sowie um die Verordnung (EU) Nr. 1177/2011 des Rates zur Änderung der VO (EG) Nr. 1467/97 über die Beschleunigung und Klärung des Verfahrens bei einem übermäßigen Defizit, AB1. 2011 Nr. L 306/33 und die Richtlinie 2011/85/EU des Rates über die Anforderungen an die haushaltspolitischen Rahmen der Mitgliedstaaten, jeweils v. 8.11.2011, AB1. 2011 Nr. L 306/33.

15 Es handelt sich um den Vorschlag für eine Verordnung des Europäischen Parlaments und des Rates über den Ausbau der wirtschafts- und haushaltspolitischen Überwachung von Mitgliedstaaten, die von gravierenden Schwierigkeiten in Bezug auf ihre finanzielle Stabilität im Euro-Währungsgebiet betroffen oder bedroht sind, KOM (2011) 819 v. 23.11.2011 sowie um das Grünbuch über die Durchführbarkeit der Einführung von Stabilitätsanleihen, KOM (2011) 818 v. 23.11.2011 - alternativ zum Begriff, ,Stabilitätsanleihen“ kann auch von „Euro-Bonds“ gesprochen werden, es wird in diesem Zusammenhang auch von der two-pack initiative gesprochen. 
Standards zum einen „konstitutionalisieren“, d.h. der Änderung im Rahmen ordentlicher Gesetzgebung entziehen, und zum anderen deren Verpflichtungsniveau hin auf eine Stabilitätsunion noch steigern.

Die Substanz des Fiskalvertrages ist nachfolgend - schon weil es Denkwürdiges zu entdecken gibt - näher in den Blick zu nehmen (II.). Anschließend wird nach dem Zusammenhang zwischen der Normativität der europäischen Rechtsgemeinschaft und dem Ausweichen einer Mehrheit von Mitgliedstaaten auf die intergouvernementale Ebene gefragt (III.). Die Folgen für die Idee der europäischen Integration stehen im Mittelpunkt des viertens Abschnitts (IV.), an den sich eine Diskussion der Auswirkungen auf das deutsche Verfassungsgefüge anschließt (V.). Der Schluss unternimmt eine kurze Gesamtwürdigung (VI.).

\section{Substanz des Fiskalvertrages}

\section{Verhandlungsrahmen}

Der Fiskalvertrag ist das Ergebnis europäischer Kabinettsdiplomatie. Nachdem der Dissens in der EU-27 über eine förmliche Primärrechtsänderung Anfang Dezember 2011 zu Tage getreten war, konnten die Mitgliedstaaten für den nunmehr angestrebten intergouvernementalen Vertrag sogar auf einen „kleinen Konvent" verzichten. ${ }^{16}$ Der Vertragstext entstand aus den Verhandlungen einer Arbeitsgruppe „Fiskalpolitische Stabilitätsunion“, an der auf Einladung des Präsidenten des Europäischen Rates Vertreter aller 27 Mitgliedstaaten und drei Abgeordnete des Europäischen Parlaments ${ }^{17}$ unter Vorsitz des luxemburgischen Finanzstaatssekretärs teilnahmen.

Der Verhandlungsfortgang spiegelt sich in fünf Vertragsentwürfen, die der Arbeitsgruppe zwischen dem 16. Dezember 2011 und dem 27. Januar 2012 - innerhalb von fünf Wochen - vorgelegt wurden. Die Endfassung des Vertrages enthält neben redaktionellen Anpassungen auch weitere materielle Änderungen. Obwohl der Fiskalvertrag von Regierungsvertretern ausgehandelt wurde, gelangten die

16 Im vereinfachten Änderungsverfahren nach Art. 48 Abs. 6 und 7 EUV kann auf die Einberufung eines Konvents aus Vertretern der nationalen Parlamente, des Europäischen Parlaments, der Kommission und der Staats- und Regierungschefs der Mitgliedstaaten (Art. 48 Abs. 3 EUV) verzichtet werden; vgl. auch das Schreiben des Auswärtigen Amtes, a.a.O., 2011, 5.

17 Bei den drei Mitgliedern des Europäischen Parlaments handelte es sich um Elmar Brok (EPP, DE), Roberto Gualtieri (S\&D, IT) und Guy Verhofstadt (ALDE, BE); zur kritischen Position des Parlaments s. Entschließung v. 18.1.2012 zu den Schlussfolgerungen der Tagung des Europäischen Rats (8.9. Dezember 2011) zum Entwurf eines Internationalen Abkommens über eine verstärkte Wirtschaftsunion (P7 TA(2012)0002). 
Entwurfsfassungen, teilweise informell, über das Internet an die Öffentlichkeit. Anhand einer Gegenüberstellung der Entwurfsfassungen ${ }^{18}$ kann vor dem Hintergrund des politischen Kontextes, der Änderungsvorschläge der drei EuropaParlamentarier ${ }^{19}$ und des deutschen Strategiepapiers der Verhandlungsverlauf gut nachvollzogen werden. Es kommt zu dem paradoxen Ergebnis, dass der Fiskalvertrag trotz des nicht einberufenen Konvents ein transparenter primärrechtsgleicher Akt ist.

\section{Kernregelungen}

a) Defizitgrenze-Schuldenbremse - , 1/20-Regelung“

Das Herz des Fiskalvertrages schlägt in seinem Titel III. Er stellt der Überschrift nach den eigentlichen „Fiskalpolitischen Pakt“ dar. Die Vertragsparteien verpflichten sich, zusätzlich zu ihren Vertragspflichten aus dem Unionsrecht:

- den gesamtstaatlichen Haushalt auszugleichen oder einen Überschuss auszuweisen (Art. 3 Abs. 1 lit. a FV),

- bei Nichterreichung dieses Ziels entsprechende fiskalpolitische Korrekturmaßnahmen innerhalb eines bestimmten Zeitraums zu ergreifen (Art. 3 Abs. 1 lit. e FV),

- die Defizitregeln und den Korrekturmechanismus innerhalb eines Jahres nach Inkrafttreten des Fiskalvertrages in verbindlicher und dauerhafter Rechtsform, „vorzugsweise mit Verfassungsrang“, zu verankern (Art. 3 Abs. 2 FV),

- eine über dem Schwellenwert von 60 Prozent liegende gesamtstaatliche Verschuldung um jährlich durchschnittlich ein Zwanzigstel (,1/20Regelung“) zu verringern $^{20}$ (Art. 4 FV),

- $\quad$ im Fall eines Defizitverfahrens dem Rat und der Kommission eine detaillierte Beschreibung des zu beschließenden und umzusetzenden Strukturprogramms zur Genehmigung vorzulegen (Art. 5 FV),

18 Eine Synopse der fünf englischsprachigen Entwurfsfassungen und des endgültigen Vertragstextes ist im Internet unter http://www.zse.nomos.de/synopse-fiskalvertrag.pdf einsehbar. Die Synopse ermöglicht einen ertragreichen analytischen Blick auf die Entstehung des Fiskalvertrages und liefert tragfähige Argumente für dessen Auslegung und Anwendung. Nach dem Inkrafttreten werden die Amtssprachen der beteiligten Mitgliedstaaten jeweils authentische Vertragssprachen sein, s. Art. 16 FV.

19 European Parliament Representatives Amendments, Dezember 2011, vgl. auch Ziff. 4 der EntschlieBung vom 18.1.2012 (Fn. 17).

20 Die Pflicht zum Schuldenabbau ist nicht sofort nach Inkrafttreten des Fiskalvertrages, sondern nach der dreijährigen Übergangsfrist des Art. 2 Abs. 1a VO (EG) Nr. 1467/97 idF der VO 1177/2011 anwendbar, vgl. die Antwort der Bundesregierung auf die Frage des Abgeordneten Alexander Ulrich, BT-Drs. 17/8637, Nr. 58, 38 . 
- den Rat und die Kommission über die geplante Begebung von Staatsschuldtiteln im Voraus zu unterrichten (Art. 6 FV) und

- der Kommission in einem Defizitverfahren gegen einen Euro-Staat im Hinblick auf Vorschläge und Empfehlungen grundsätzlich zuzustimmen, es sei denn, es findet sich eine qualifizierte Mehrheit gegen den vorgeschlagenen oder empfohlenen Beschluss (Art. 7 FV).

Die Goldene Regel eines Haushaltsausgleichs (Art. 3 Abs. 1 lit. a FV) gilt als eingehalten, wenn - verkürzt ausgedrückt - ein jährliches strukturelles Defizit unterhalb von 0,5 Prozent des Bruttoinlandsprodukts (BIP) eingehalten wird. Die Regelung übererfüllt die politischen Vorgaben vom 9. Dezember 2011. ${ }^{21}$ Von der Regel darf unter „außergewöhnlichen Umständen vorübergehend“ abgewichen werden, wobei diese Umstände, die als Tatbestandsmerkmal ,außergewöhnliche Ereignisse" in Art. 122 Abs. 2 AEUV bei den Griechenlandhilfen eine wichtige Rolle spielten, ${ }^{22}$ vom Fiskalvertrag definiert werden. ${ }^{23}$

Die Kernregelungen des Fiskalvertrages nehmen in der Begriffswahl und durch ausdrückliche Hinweise auf den geänderten Stabilitäts- und Wachstumspakt Bezug. Zentral sind dabei vor allem das „länderspezifische mittelfristige Ziel“ und der „Anpassungspfad“. Nach der präventiven Säule des Stabilitäts- und Wachstumspaktes müssen die Mitgliedstaaten ein mittelfristiges Haushaltsziel festlegen. Es soll helfen, die Tragfähigkeit der öffentlichen Finanzen oder zu-

21 Europäischer Rat: Erklärung der Staats- und Regierungschefs des Eurowährungsgebiets v. 9.12.2011, Ziff. 4, 1. Spiegelstr., wonach ,das jährliche strukturelle Defizit generell 0,5 Prozent des nominellen BIP nicht übersteigt“ (not exceeding), was im vierten Vertragsentwurf durch die schärfere Formulierung ,mit einer Untergrenze von" (a lower limit of) ersetzt wurde.

$22 \mathrm{Zu}$ dieser Debatte Hentschelmann, K.: Finanzhilfen im Lichte der No-Bailout-Klausel, in: Europarecht 46 (2011), 282-312; Wieland, J.: Der Rettungsschirm für Irland, in: Neue Zeitschrift für Verwaltungsrecht 30 (2011), 340-343, hier 342; Herrmann, C.: Griechische Tragödie - der währungsverfassungsrechtliche Rahmen für die Rettung, den Austritt oder den Ausschluss von überschuldeten Staaten aus der Eurozone, in: Europäische Zeitschrift für Wirtschaftsrecht 21 (2010), 413-418, spez. 415; Calliess, C.: Perspektive des Euro zwischen Solidarität und Recht, in: Zeitschrift für europarechtliche Studien (2011), 213-282, hier 268; Louis, J.-V.: Guest Editorial: The No-bailout Clause and Rescue Packages, Common Market Law Review 47 (2010), 971-986; Schröder, U. J.: Die Griechenlandhilfen im Falle ihrer Unionsrechtswidrigkeit, in: Die öffentliche Verwaltung 64 (2011), 61-68; Hufeld, U.: Zwischen Notrettung und Rütlischwur, in: integration 34 (2011), 117-131.

23 Art. 3 Abs. 3 lit. b FV: „'Außergewöhnliche Umstände’ sind ein außergewöhnliches Ereignis, das sich der Kontrolle der betreffenden Vertragspartei entzieht und erhebliche Auswirkungen auf die Lage der öffentlichen Finanzen hat, oder ein schwerer Konjunkturabschwung im Sinne des geänderten Stabilitätsund Wachstumspakts, vorausgesetzt, die vorübergehende Abweichung der betreffenden Vertragspartei gefährdet nicht die mittelfristige Tragfähigkeit der öffentlichen Finanzen.“; s. bereits Art. 5 Abs. 1 UAbs. 10 VO (EG) Nr. 1466/97 idF der VO 1175/2011. 
mindest eine rasche Annäherung an diesen Zustand zu gewährleisten. ${ }^{24}$ Diese länderspezifischen mittelfristigen Haushaltsziele sollen sich innerhalb einer konkreten Spanne bewegen, die konjunkturbereinigt und ohne Anrechnung einmaliger und befristeter Maßnahmen zwischen -1 Prozent des BIP und einem zumindest ausgeglichenen Haushalt liegt. ${ }^{25}$ Aus diesem Seitenblick auf den Stabilitätsund Wachstumspakt wird deutlich, dass der Fiskalvertrag die fiskalpolitischen Anforderungen an die Mitgliedstaaten verdoppelt hat - vom mittelfristigen Haushaltsziel darf jährlich nicht mehr um 1 Prozent, sondern nur noch bis zu 0,5 Prozent abgewichen werden (Art. 3 Abs. 1 lit. b FV). ${ }^{26}$

\section{b) Wirtschaftsregierung - Koordinierung und Konvergenz}

In der Erklärung vom 9. Dezember 2011 verpflichteten sich die Staats- und Regierungschefs, auf eine gemeinsame Wirtschaftspolitik hinzuarbeiten. Zu diesem Zweck sollte ein Verfahren eingeführt werden, das sicherstellt, dass alle wichtigen wirtschaftspolitischen Reformpläne der Euro-Staaten auf Ebene des EuroWährungsgebiets erörtert und abgestimmt werden, um ,benchmarks für vorbildliche Vorgehensweisen festzulegen““. ${ }^{27}$ Art. 9 und 11 FV setzen diese Verpflichtung mit demselben Grad an Verbindlichkeit um (,verpflichten sich [...], gemeinsam auf eine Wirtschaftspolitik hinzuarbeiten“). Um vorbildliche Maßstäbe festzulegen, werden die Vertragsparteien ,größere wirtschaftspolitische Reformen“" vorab erörtern und unter Umständen auch koordinieren. ${ }^{28}$

Es wird ausdrücklich auf das Instrument der verstärkten Zusammenarbeit verwiesen, ${ }^{29}$ von dem die Vertragsparteien zwecks reibungslosem Funktionieren des Währungsgebiets ,aktiven Gebrauch“ machen wollen (Art. $10 \mathrm{FV}$ ). Dabei fällt

24 Ergänzend müssen die Mitgliedstaaten so genannte Numerische Haushaltsregeln i.S. einer mittelfristigen Finanzplanung in ihr nationales Recht aufnehmen, s. Art. 5 bis 7 RL 2011/85/EG.

25 Art. 2 a VO (EG) Nr. 1466/77 i.d.F. der VO (EG) Nr. 1175/2011.

26 Vgl. die Antwort der Bundesregierung auf die Frage des Abgeordneten Alexander Ulrich, BT-Drs. 17/8637, Nr. 59, 39-40.

27 Europäischer Rat: Erklärung der Staats- und Regierungschefs des Eurowährungsgebiets vom 9.12.2011, Ziff. 9.

28 Bei dieser Formulierung könnte an die zwischen Deutschland und Frankreich bereits besprochene Harmonisierung der Unternehmenssteuern und der Bemessungsgrundlagen sowie an die Einführung neuer Steuern, wie etwa einer Finanztransaktionssteuer, aber auch an Reformen des Arbeitsmarktes und der Rentensysteme gedacht sein, vgl. Gemeinsamer Deutsch-Französischer Brief an EU-Ratspräsident Herman Van Rompuy, a.a.O., 2011.

29 S. Art. 20, 326 bis 334 AEUV; ein Hinweis auf die Verstärkte Zusammenarbeit ist bereits enthalten in dem Gemeinsamen Deutsch-Französischen Brief an EU-Ratspräsident Herman Van Rompuy, a.a.O., 2011 . 
auf, dass der Binnenmarkt ausdrücklich hervorgehoben wird. Er dürfe nicht beeinträchtigt werden, während der von Art. 326 Abs. 2 AEUV gleichfalls genannte wirtschaftliche, soziale und territoriale Zusammenhalt sowie der Schutz vor Wettbewerbsverzerrungen und Diskriminierungen nicht eigens betont werden.

Die Aussagen in diesem Titel IV des Fiskalvertrages scheinen sich auf den ersten Blick im üblichen Rahmen der wirtschaftspolitischen Koordinierung zu halten. ${ }^{30}$ Die Verhandlungsgeschichte zeigt jedoch, dass die Delegationen um einzelne Formulierungen und Wörter gerungen haben. Aus einer Gesamtschau lässt sich sagen, dass die endgültige Vertragsfassung den zu intensiven Anschein eines Ziels gleichmäßiger Wirtschaftstätigkeit im Sinne wirtschaftlicher Konvergenz vermeiden soll. Die wechselnden Überschriften des Titels IV zeigen das Ringen zwischen „Konvergenz“ und „Koordinierung“; zugleich sprach erstmals der dritte Entwurf von einer „gemeinsamen“ Wirtschaftspolitik (common economic policy), was im fünften Entwurf wieder zugunsten einer Wirtschaftspolitik gestrichen wurde. Der bereits im ersten Entwurf enthaltene Verweis auf den EuroPlus-Pakt ist seit dem vierten Entwurf gestrichen, so dass nunmehr nur der 24. Erwägungsgrund der Präambel auf dieses wirtschaftspolitische Instrument hinweist. Die durchgängige Ersetzung des Adjektivs gleichmäßig (smooth) durch reibungslos (proper) passt ebenfalls in dieses Bild.

\section{c) Verfahren - ,umgekehrte qualifizierte Mehrheit“}

Besondere Aufmerksamkeit verdienen die Verfahrensregeln des Fiskalvertrages. Ein Schwachpunkt des ursprünglichen Stabilitäts- und Wachstumspaktes bestand in der Notwendigkeit seitens der Euro-Staaten, über Defizitverstöße und Sanktionen mit qualifizierter Mehrheit positive Beschlüsse fassen zu müssen. Es war deshalb bereits ein Ziel der sekundärrechtlichen Änderungen des Stabilitäts- und Wachstumspaktes, mit einem mitgliedstaatlichen Fehlverhalten automatisch Konsequenzen zu verbinden. Die Annahme des so genannten six-pack ${ }^{31}$ verzögerte sich aufgrund eben dieser Frage, weil das Europäische Parlament und der Rat zunächst keinen Konsens fanden. Das Parlament bevorzugte, offenbar nach Vorbild der WTO-Streitschlichtung, eine „umgekehrte qualifizierte Mehrheit“. Nach dieser Idee müsste die Nichtverhängung von Sanktionen mit qualifizierter

30 Einen neueren Überblick gibt Seidel, M.: Eine Wirtschaftsregierung für die Europäische Union, in: Müller-Graff/Schmahl/Skouris (Hg.): Festschrift für Dieter H. Scheuing, Baden-Baden, 2011, 717-734.

31 Vgl. unter Fn. 14. 
Mehrheit vom Rat beschlossen werden. Der Rat der Wirtschafts- und Finanzminister lehnte den damit verbundenen Automatismus ab. Der europäische Gesetzgeber fand dann Ende September/Anfang Oktober 2011 einen Kompromiss, der an die letzte Stufe des so genannten Regelungsverfahrens bei der Durchführungsrechtsetzung (Komitologie) erinnert. ${ }^{32}$

Der Rat kann eine Empfehlung der Kommission mit qualifizierter Mehrheit annehmen, nach der ein Mitgliedstaat von seinem mittelfristigen Haushaltsziel oder dem Anpassungspfad dorthin abweicht. Trifft der Rat diese Entscheidung nicht und sieht der betreffende Mitgliedstaat weiterhin keine geeigneten Abhilfemaßnahmen vor, unterbreitet die Kommission nach einem Monat erneut die Empfehlung. Sie gilt als angenommen, wenn der Rat die Empfehlung nicht innerhalb von zehn Tagen mit einfacher Mehrheit ablehnt. ${ }^{33}$ Allerdings ist der Kompromiss nur auf die Nichteinhaltung der Stabilitätsprogramme in der präventiven Säule des Stabilitäts- und Wachstumspaktes bezogen.

Was bei der Würdigung des Kompromisses aber kaum beachtet wird, ist die direkte Verwirklichung einer umgekehrten qualifizierten Mehrheit in der korrektiven Säule des Stabilitäts- und Wachstumspaktes. In dem Verfahren über die wirksame haushaltspolitische Überwachung gelten Sanktionsbeschlüsse über Einlagen oder Geldbußen als angenommen, wenn der Rat die entsprechenden Empfehlungen der Kommission nicht innerhalb von zehn Tagen, nachdem die Kommission sie angenommen hat, mit qualifizierter Mehrheit ablehnt. ${ }^{34}$

Mit dem Fiskalvertrag verpflichten sich die Vertragsparteien, die Empfehlungen und Vorschläge der Kommission zu unterstützen, allerdings nur für den Fall, dass das Defizit-Kriterium verletzt ist (Art. 7 FV). ${ }^{35}$ Die Regelung, die seit dem

32 S. Rat der EU: Pressemitteilung v. 4.10.2011, Nr. 14988/11, Annex, 5: Die Zauberformel heißt: „Automaticity in the voting procedure“. Zur Parallele mit dem Regelungsverfahren vgl. Art. 5 Abs. 6 Beschluss des Rates 1999/468/EG v. 28.6.1999 zur Festlegung der Modalitäten für die Ausübung der der Kommission übertragenen Durchführungsbefugnisse (Komitologie-Beschluss) i.d.F. des Beschlusses des Rates 2006/512/EG vom 17.6.2006.

33 Art. 121 Abs. 4 AEUV i.V.m. Art. 6 Abs. 2 UAbs. 4 und 5 VO (EG) Nr. 1466/97 idF der VO (EG) Nr. 1175/2011.

34 Art. 4 Abs. 2, 5 Abs. 2 und Art. 6 Abs. 2 VO (EG) Nr. 1173/2011. Für die Berechnung der qualifizierten Mehrheit gilt Art. 238 Abs. 3 lit. b AEUV; abstimmungsberechtigt sind nur die Vertreter von EuroStaaten, die Stimmen des Vertreters aus dem betreffenden Mitgliedstaat werden nach Art. 12 VO (EG) Nr. 1173/2011 nicht berücksichtigt.

35 Art. 7 FV ist nicht anwendbar auf die präventive Säule des Stabilitäts- und Wachstumspaktes (Art. 6 Abs. 2 und Art. 10 Abs. 2 VO (EG) Nr. 1466/97 i.d.F. der VO (EG) Nr. 1175/2011), auf Regeln über die Vermeidung und Korrektur makroökonomischer Ungleichgewichte (VO (EG) Nr. 1174/2011 und VO (EG) Nr. 1176/2011) sowie auf das Schuldenkriterium und Sanktionen bei Manipulationen von Statisti- 
vierten Vertragsentwurf bindend ausgestaltet wurde,${ }^{36}$ ist eine mitgliedstaatliche Vorfestlegung auf das Abstimmungsverhalten. ${ }^{37}$ Zugleich wird auf die qualifizierte (Veto-)Mehrheit hingewiesen, so dass die Regelung zirkulär erscheint. Denn halten sich alle Vertragsparteien an Art. 7 FV, dann kann es eigentlich keine Stimmen gegen die Kommission geben.

Die Regel der umgekehrten qualifizierten Mehrheit und die Festlegung auf das Abstimmungsverhalten stehen in einem bemerkenswerten politischen Kontext, der die deutsch-französische Rolle als führende Unionsmächte betont und das besondere Interesse an deren Fortbestand stützt. Denn Deutschland und Frankreich haben im Ergebnis eine Ermöglichungsminorität. Unterstützen diese beiden Mitgliedstaaten die Vorschläge oder Empfehlungen der Kommission, werden diese stets angenommen werden. Die gewogenen Stimmen der 15 anderen EuroStaaten erreichen nämlich zusammen nicht das notwendige Veto-Quorum. Das gilt allerdings nur, solange Deutschland oder Frankreich nicht selbst betroffen sind; in diesem Fall würden deren Stimmen - wie auch in allen anderen Verfahren - nicht berücksichtigt. ${ }^{38}$

\section{d) Durchsetzung und Justiziabilität}

Die Staatsschuldenkrise hat der Öffentlichkeit eine bis dahin wenig bekannte Regel der Wirtschafts- und Währungsunion bewusst gemacht: Im Defizitverfahren können weder die Kommission noch die Mitgliedstaaten ihr Klagerecht auf

ken (Art. 7 VO (EG) Nr. 1173/2011), vgl. Pernice, I.: International Agreement On A Reinforced Economic Union, Legal Opinion, 9.1.2012, $10 \mathrm{ff}$; Peers, S.: Draft Agreement on Reinforced Economic Union, Analysis, State Watch, 21.12.2011, 8.

36 Das Verb undertake wurde mit dem vierten Entwurf durch commit ersetzt; die in Fn. 35 genannten Stellungnahmen zweifeln unter Hinweis auf die Verwendung von undertake an der Verbindlichkeit von Art. 7 FV.

37 Bundesregierung: Denkschrift zum Fiskalvertrag, BR-Drs. 130/12, a.a.O., 2012, 23: verbindliche Vereinbarung über die Stimmrechtsausübung im Rat.

38 Nach Art. 7 FV ist die qualifizierte Mehrheit analog zu den einschlägigen Regeln des EUV und AEUV zu berechnen, d.h. es sind Art. 16 Abs. 5 EUV; Art. 139 Abs. 4 AEUV und Art. 3 Abs. 3 und 4 des Protokolls Nr. $36 \mathrm{zu}$ den Verträgen anwendbar. Die 17 Euro-Staaten haben zusammen 213 gewogene Stimmen. Das Quorum für die qualifizierte Mehrheit bei Abstimmungen im Rat liegt allgemein bei 74 Prozent (255 von insgesamt 345 Stimmen). Da dieser Prozentsatz auch auf Abstimmungen unter den Euro-Staaten anwendbar ist, werden 158 Stimmen benötigt ( 213 x 0,74=157,62, aufgerundet 158), um eine Empfehlung oder einen Vorschlag der Kommission zu verhindern. Deutschland und Frankreich verfügen jeweils über 29 Stimmen im Rat; wenn also die beiden Staaten einen Kommissionsvorschlag mittragen, dann können die anderen 15 Euro-Staaten maximal nur noch 155 Stimmen auf die Waage bringen - drei zu wenig. 
Vertragsverletzung ausüben. ${ }^{39}$ Das vertragliche Defizitverfahren, mit seiner sekundärrechtlichen Konkretisierung, ist insoweit das speziellere Durchsetzungsinstrument. Der Fiskalvertrag nimmt diese Thematik auf und versucht den genannten Artikel in der Sache zu ändern, indem der Rechtsweg zum EuGH begründet wird (Art. 8 FV). Das Primärrecht sieht vor, dass die Mitgliedstaaten ,jede mit dem Gegenstand der Verträge im Zusammenhang stehende Streitigkeit" zwischen ihnen im Wege eines Schiedsvertrages vor den EuGH bringen können. ${ }^{40}$

Der Gerichtshof kann nunmehr durch einen oder mehrere Vertragsparteien mit der Frage befasst werden, ob eine Vertragspartei die Pflichten aus Art. 3 Abs. 2 FV nicht erfüllt hat. ${ }^{41}$ Hat die Kommission zuvor festgestellt, dass Art. 3 Abs. 2 FV nicht erfüllt ist, besteht sogar eine Klagepflicht. Die Vertragsparteien haben in einem bislang nicht bekannt gewordenen Protokoll zum Fiskalvertrag festgelegt, dass die Klage grundsätzlich von den Mitgliedstaaten erhoben wird, die den Dreiervorsitz im Rat führen. ${ }^{42}$ Bei einer Verurteilung soll der Gerichtshof die beklagte Vertragspartei dazu verpflichten, innerhalb einer festzulegenden Frist die erforderlichen Maßnahmen zu treffen (Art. 8 Abs. 1 FV). Sollte die beklagte Vertragspartei weiter säumig bleiben, kann der EuGH in einem zweiten Schritt erneut angerufen werden, um die Verhängung finanzieller Sanktionen in Form eines Pauschalbetrages oder eines Zwangsgeldes $\mathrm{zu}$ beantragen (Art. 8 Abs. 2 FV). ${ }^{43}$

Mit dieser begrenzten Justiziabilität wird noch einmal die Grundkonzeption des Fiskalvertrages deutlich: Die Vertragsparteien verpflichten sich, die Defizitgren-

39 Art. 126 Abs. 10 AEUV.

40 S. die ausdrückliche Inanspruchnahme des Art. 273 AEUV durch Art. 8 Abs. 3 FV, womit dem Schriftformerfordernis aus Art. $38 \S 6 \mathrm{VerfO} / \mathrm{EuGH}$ Genüge getan wird; vgl. auch den 15. Erwägungsgrund der Präambel des Fiskalvertrages, der auf den Schiedsvertrag ausdrücklich hinweist.

41 Die zweite Entwurfsfassung erweiterte die Justiziabilität auf den gesamten Titel III des Fiskalvertrages, was allerdings bereits im dritten Entwurf wieder zurückgenommen wurde.

42 Protokoll über die Unterzeichnung des Vertrags über Stabilität, Koordinierung und Steuerung in der Wirtschafts- und Währungsunion v. 2.3.2012, BR-Drs. 130/12, a.a.O., 2012, 18-19. Die Reihenfolge der Dreiervorsitze ist geregelt im Beschluss 2009/908/EG des Rates v. 1.12.2009, AB1. 2009 Nr. L 344/28, 56. Die Klage muss innerhalb von drei Monaten nach Eingang des Kommissionsberichts bei den Vertragsparteien eingereicht werden.

43 S. Art. 260 AEUV; der 16. Erwägungsgrund der Präambel des Fiskalvertrages verweist in diesem Zusammenhang indirekt auf die Europäische Kommission: Mitteilung - Anwendung von Artikel 228 EG-Vertrag (SEK (2005) 1658). Die Sanktion soll nicht über 0,1 Prozent des BIP hinaus verhängt werden. Die Verhängung finanzieller Sanktionen ist durchaus noch von Art. 273 AEUV gedeckt, der grundsätzlich auf das anwendbare Unionsrecht, d.h. auch Art. 260 AEUV Bezug nimmt, vgl. Karpenstein, U.: Art. 273 in: Grabitz/Hilf/Nettesheim (Hg.): Recht der EU, 45. Lieferung, Rn. 15-16. 
ze und einen Korrekturmechanismus in ihr nationales Recht aufzunehmen, das sodann den unmittelbaren Maßstab für das jeweilige mitgliedstaatliche Handeln bildet. Art. 3 Abs. 1 und 2 FV sind im Grunde wie eine Richtlinie des Sekundärrechts konstruiert. Bei einer am Wortlaut orientierten Auslegung begrenzt sich die Justiziabilität auf die bloße Umsetzung, nicht aber auf die Einhaltung der materiellen Regeln des Art. 3 Abs. 1 FV. Die Bundesregierung vertritt in ihrer Denkschrift zum Vertrag diese Auslegung. Sinn und Zweck des Fiskalvertrages sprechen aber dafür, dass der EuGH auch prüfen muss, ob die Defizitgrenze und der Korrekturmechanismus in den jeweiligen Haushaltsjahren eingehalten wurden. Die Justiziabilität würde sich anderenfalls auf eine einmalige Prüfung der nationalen Rechtslage beschränken. Für die umfangreichere Zuständigkeit spricht auch, dass die bis zur zweiten Entwurfsfassung vorgesehene Jurisdiktion nationaler Gerichte über die Anwendung (implementation) des Umsetzungsrechts seit dem dritten Entwurf nicht mehr vorgesehen war. Gegen diese Auslegung spricht der bereits erwähnte Art. 126 Abs. 10 AEUV. Der Fiskalvertrag will einen primärrechtlichen Widerspruch ausdrücklich vermeiden. ${ }^{44}$

Bei der Justiziabilität der Defizitgrenze hat die Kommission eine testierende Funktion. Sie legt einen Bericht über die Einhaltung des Art. 3 Abs. 2 FV vor und beurteilt das Handeln einer bereits verurteilten Vertragspartei. Ein eigenes Klagerecht, das ihr als „Hüterin der Verträge“ nach dem AEU-Vertrag zusteht, hat sie nicht erhalten. Dieser Schritt hätte wiederum Art. 126 Abs. 10 AEUV widersprochen. Das lenkt die Aufmerksamkeit darauf, dass Kommission und Gerichtshof von den Parteien des Fiskalpaktes als Dienstleister ausgeliehen werden. Während Art. 273 AEUV diese Organleihe des Gerichtshofes in das Ermessen der Mitgliedstaaten stellt, ist eine entsprechende Indienstnahme der Kommission nur möglich, wenn alle Mitgliedstaaten zustimmen. ${ }^{45}$ Der Fiskalvertrag bemüht sich deshalb um die Kongruenz des Kommissionshandelns mit den Handlungsmöglichkeiten aus Titel VIII des AEU-Vertrages.

44 S. Art. 2 Abs. 2 FV und nachfolgend unter II.3.

45 S. den Beschluss der Vertreter der Regierungen der 27 EU-Mitgliedstaaten v. 9.5.2010: „Die 27 Mitgliedstaaten kommen überein, dass es der Kommission gestattet sein wird, in diesem Zusammenhang von den Mitgliedstaaten des Euro-Währungsgebiets beauftragt zu werden." Rat der EU, Dok. Nr. 9614/10, Anlage. Bereits aus der Existenz dieses Beschlusses durch alle 27 Mitgliedstaaten wird im Umkehrschluss deutlich, dass die Indienstnahme der Kommission durch den Fiskalvertrag über die bestehenden Aufgaben hinaus ohne die Zustimmung Großbritanniens und Tschechiens nicht möglich ist. 


\section{Verzahnungen}

\section{a) Verzahnung mit dem Recht der Europäischen Union}

Auf dem Fiskalvertrag liegt der Schatten, dass er die Alternative zu der politisch angestrebten, aber nicht erreichbaren Änderung des Primärrechts ist. So liegt die Kritik nahe, dass das ordentliche Vertragsänderungsverfahren im EU-Vertrag, das einstimmiges Handeln der Mitgliedstaaten anordnet, umgangen wurde. Einzelne Vertragsregelungen nehmen das Thema auf und versuchen es eindeutig, im Sinn der Integrität des Unionsrechts, zu lösen. Eine salvatorische Klausel erklärt den Fiskalvertrag nur insoweit für anwendbar, wie er mit dem Primär- und Sekundärrecht vereinbar ist (Art. 2 Abs. 2 FV). Ausdrücklich verpflichten sich die Vertragsparteien, den Fiskalvertrag in einer mit dem Gebot der loyalen Zusammenarbeit vereinbaren Weise anzuwenden und auszulegen. ${ }^{46}$ Der noch in der zweiten Entwurfsfassung enthaltene ausdrückliche Hinweis auf den Anwendungsvorrang des Unionsrechts gegenüber dem Fiskalvertrag wurde mit dem dritten Entwurf gestrichen.

Dass der Fiskalvertrag gleichwohl als ein Laboratorium vernünftiger Primärrechtsfortentwicklung wahrgenommen werden soll, bezeugt die Klausel, dass binnen höchstens fünf Jahren nach seinem Inkrafttreten die notwendigen Schritte unternommen werden, den Fiskalvertrag in den EU-Rechtsrahmen zu überführen (Art. 16 FV). Der asymmetrische politische Dissens unter den Mitgliedstaaten wird dadurch in den positiven Erzählstrang des Schengen-Raums gestellt, der außerhalb der Gemeinschaft gegründet und dessen acquis 1999 in den EGVertrag überführt wurde. ${ }^{47}$

Schließlich erinnert die Struktur des Fiskalvertrages an eine sekundärrechtliche Richtlinie. Die für einen völkerrechtlichen Vertrag dieses überschaubaren Umfangs ungewöhnlich lange Präambel ${ }^{48}$ ist typisch für die Erwägungsgründe von Sekundärrechtsakten. Sie ersetzen weitgehend eine Begründung des Rechtsaktes

46 Art. 2 Abs. 1 FV unter Hinweis auf Art. 4 Abs. 3 EUV. Weitere Vorbehalte zugunsten des EU-Rechts sind enthalten im 20. Erwägungsgrund der Präambel sowie in Art. 3 Abs. 1, 5 Abs. 1, 7, 10 und 11 FV.

47 Beschluss des Rates 1999/435/EG v. 20.5.1999 zur Bestimmung des Schengen-Besitzstands zwecks Festlegung der Rechtsgrundlagen für jede Bestimmung und jeden Beschluss, die diesen Besitzstand bilden, nach Maßgabe der einschlägigen Bestimmungen des Vertrags zur Gründung der Europäischen Gemeinschaft und des Vertrags über die Europäische Union, ABl. 1999 Nr. L 176/1; vgl. dazu auch die Dokumentation des Generalsekretariats des Rates, Schengen acquis, Luxemburg, 2001.

48 Die Präambel umfasst 27 Erwägungsgründe; s. auch die allein in der zweiten Entwurfsfassung um die gesamte Präambel gesetzten eckigen Klammern, die einen prinzipiellen Dissens über den eingeklammerten Text signalisieren. 
durch den Gesetzgeber. Die materiellen Vorgaben für die Defizitgrenze und den Korrekturmechanismus sind zudem innerhalb eines Jahres in nationales Recht aufzunehmen und, da dies auf der Grundlage gemeinsamer, von der Kommission vorzuschlagender Grundsätze geschehen soll, wird ein Umsetzungsverhältnis begründet. ${ }^{49}$ Im Ergebnis werden die bereits erfolgten Änderungen des Stabilitäts- und Wachstumspaktes erweitert.

\section{b) Verzahnung mit dem Europäischen Stabilitätsmechanismus (ESM)}

Eine weitere Verzahnung erfolgt mit dem Euro-Rettungsfonds und seiner institutionellen Verstetigung, dem Europäischen Stabilitätsmechanismus (ESM). Mit dem ESM wird der Rettungsfonds auf eine völkervertragliche Grundlage gestellt. Seine Tätigkeit als selbständige internationale Organisation neben der EU und der EZB - ein gutes Stück weit nach dem Vorbild des Internationalen Währungsfonds gestaltet - wird unbefristet sein. Das Rettungsinstrumentarium, vorsorgliche Finanzhilfen, Darlehen, Rekapitalisierung von Finanzinstituten und Monetarisierung von Staatsschulden durch Anleihekäufe auf dem Primär- oder Sekundärmarkt, entspricht dem der ertüchtigten Europäischen Finanzstabilisierungs-Fazilität (EFSF), kann aber erweitert werden. ${ }^{50}$

Die Präambel des Fiskalvertrages lässt keinen Zweifel daran, dass EuroSchuldnerstaaten sich ab dem 1. März 2013 nur dann aus dem ESM neu finanzieren können, wenn sie zuvor den Fiskalvertrag ratifiziert und die Pflicht aus Art. 3 Abs. 2 FV erfüllt haben. ${ }^{51}$ Nach der Präambel achtet der Fiskalvertrag zudem bestehende wirtschaftspolitische Auflagen gegenüber Schuldnerstaaten, die von Europäischer Union, dem Internationalen Währungsfonds und von Mitgliedstaaten - hier schlagen die bilateralen Kredite für Griechenland zu Buche - gemacht wurden. Aufgrund der Anwendung des Fiskalvertrages sollen diese nicht geändert werden. ${ }^{52}$

49 Vgl. Funke, A.: Umsetzungsrecht, Tübingen, 2010, 156-223.

50 Die Finanzhilfefazilitäten sind in Art. 14 bis 18 ESM-Vertrages geregelt, die „Überprüfung“ seitens des Gouverneursrates wird durch Art. 19 ermöglicht. Eine Würdigung des letzten ESM-Vertragsentwurfs jüngst bei Kube, $H .:$ Rechtsfragen der völkerrechtlichen Euro-Rettung, in: Wertpapier-Mitteilungen 66 (2012), 245-253, hier 245-247.

51 25. Erwägungsgrund der Präambel FV. Der Bedingungszusammenhang zwischen Finanzierung und Einhaltung des Art. 3 Abs. 2 FV wurde in der dritten Entwurfsfassung, zwischen Finanzierung und Ratifikation in der vierten Entwurfsfassung des Fiskalvertrages aufgenommen.

52 20. Erwägungsgrund der Präambel FV, der mit der dritten Entwurfsfassung in den Vertragstext aufgenommen wurde. 


\section{Normativität des alten und neuen Vertragsrechts}

Welcher Zusammenhang besteht nun zwischen dem Fiskalvertrag und der Normativität der europäischen Rechtsgemeinschaft? Der Fiskalvertrag ist ein Instrument des Rechts. Die Haushalte der Vertragsparteien sollen in Zukunft annähernd ausgeglichen, ihre öffentlichen Finanzen dauerhaft tragfähig sein, beides soll Bürger, Unternehmen und andere Staaten davon überzeugen, dass sie den beteiligten Euro-Staaten weiter vertrauen oder ihnen neues Vertrauen schenken können. ${ }^{53}$ Zwar bestehen mitgliedstaatliche Pflichten, die der Fiskalvertrag enthält, seit Dezember 2011 bereits auf der Ebene des Sekundärrechts. Der Vertrag geht jedoch, wie im voran stehenden Abschnitt skizziert, teilweise darüber hinaus und sieht eine strengere Durchsetzung vor. Die von einzelnen Mitgliedstaaten geäußerte Ansicht, dass der vertragliche Regelungsinhalt auch ohne Primärrechtsänderung, im Wege des Sekundärrechts, durch Auslegung oder durch konsensuales Handeln formlos hätte verwirklicht werden können, missachtet die Politik begrenzende Funktion des Rechts. Dem vertragsförmlichen Handeln der Mitgliedstaaten ist deshalb grundsätzlich zuzustimmen.

Doch in der vertraglichen Rechtsetzung durch eine Mehrheit von Mitgliedstaaten steckt zugleich ein schwerwiegendes Problem. Der Fiskalvertrag steht nämlich für ein Ausweichen von 25 EU-Mitgliedstaaten in die so genannte Intergouvernementalität, d.h. auf die zwischenstaatliche Regelungs- und Politikebene, die wieder einstimmiges Entscheiden außerhalb des institutionellen Rahmens der Supranationalität ermöglicht. Das Primärrecht enthält in juristischer Sprache die Bedingungen für den geldpolitischen Konsens der Mitgliedstaaten. ${ }^{54}$ Die Regeln über die Vertragsänderung (Art. 48 EUV) garantieren, dass dieser schriftliche Konsens nur in einem streng formalisierten Verfahren und einstimmig geändert werden kann. Das Vertrauen in dieses formalisierte Verfahren und die Einstimmigkeit ermöglicht es den nationalen Parlamenten überhaupt erst, durch die Vertragsratifikation die Verantwortung für das überstaatlich Ausgehandelte zu übernehmen. Nur der Vertragswortlaut kann die Grundlage sein,

$53 \mathrm{Zu}$ den ökonomischen Erscheinungsformen des gegenwärtigen Vertrauensverlustes s. Sachverständigenrat: Verantwortung für Europa wahrnehmen, Jahresgutachten 2011/12, 78-109; für den Zusammenhang von Vertrauen und Recht Luhmann, N.: Vertrauen, 2000, 41-47; Schmidt-Aßmann, E./Dimitropoulos, G.: Vertrauen in und durch Recht, in: Weingardt (Hg.): Vertrauen in der Krise, 2011, 129-149; Schaal, G. S.: Vertrauen, Verfassung und Demokratie, 2004, 75-144.

54 Die maßgeblichen Dokumente zur Entstehung des Euro sind abgedruckt in: Krägenau, H./Wetter, W. (Hg.): Europäische Wirtschafts- und Währungsunion, 1993; zu der zwiespältigen Verknüpfung von Währungsunion und deutscher Einheit Marsh, D.: The Euro, New Haven, 2011, 138-181. 
die Integrationsverantwortung wahrzunehmen und gegenüber der Öffentlichkeit zu rechtfertigen. ${ }^{55}$

Großbritannien und die Tschechische Republik stehen außen vor und müssen zur Kenntnis nehmen, dass die von ihnen abgelehnte politische Lösung nunmehr als intergouvernementaler Vertrag außerhalb des dafür eigens vorgesehenen Instruments der Verstärkten Zusammenarbeit, ${ }^{56}$ mithin außerhalb des Primärrechts verwirklicht wird. Zugleich ist es eines der Vertragsziele, die Regelungsinhalte zum neuen fiskalpolitischen Konsens der beteiligten Mitgliedstaaten zu machen und der Sekundärrechtsetzung zu entziehen. Könnte das Einstimmigkeitsversprechen des Art. 48 EUV dann auch in anderen Fällen von nur begrenztem Wert sein?

Ein zweites Problem besteht darin, dass die europäische Rechtsgemeinschaft in der Staatsschuldenkrise an ihre Grenzen stößt. ${ }^{57}$ Die Europäische Union ist auf die Folgefähigkeit und Folgebereitschaft ihrer Mitgliedstaaten angewiesen. Die Folgefähigkeit der Mitgliedstaaten beruht auf deren Verwaltungskapazität. Im Fall Griechenlands sind die diesbezüglichen Schwächen offenkundig, nicht nur im Rahmen der Steuer- und Finanzverwaltung, sondern auch bei der Grenzsicherung $^{58}$ und im Asylsystem. ${ }^{59}$ Mit Blick auf die eingeschränkte Bereitschaft, das geltende Unionsrecht anzuwenden, darf trotz aller Sympathie für die Ziele des

55 Zur kritischen Öffentlichkeit im Zusammenhang mit der Euro-Einführung Noelle-Neumann, E./Petersen, T.: Die öffentliche Meinung, Jahrbuch der europäischen Integration 1997/98, 295-302 mit einer Zusammenfassung der demoskopischen Daten der 1990er Jahre zum Euro; die deutsche politische Debatte zusammenfassend Risse, T.: Deutsche Identität und Außenpolitik, in: Schmidt/Hellmann/Wolf (Hg): Handbuch zur deutschen Außenpolitik, Wiesbaden, 2007, 56-57.

56 Art. 20 EUV, Art. 326 bis 334 AEUV; der Fiskalvertrag verweist ausdrücklich auf dieses Instrument, 22. Erwägungsgrund der Präambel und Art. $10 \mathrm{FV}$, beantwortet aber auch nicht die Frage, weshalb nicht bereits der Vertragsinhalt darauf gestützt wurde.

57 Näher dazu bereits Schorkopf, F.: Gestaltung mit Recht, in: Archiv des öffentlichen Rechts 136 (2011), 323-344.

58 Europäischer Rat: Schlussfolgerungen der Tagung vom 23./24.6.2011, EUCO 23/1/11, Ziff. 20-30 und Rat für Inneres und Justiz: Pressemittelung zur 3151. Sitzung des Rates v. 8.3.2012, Dok. Nr. 7308/12, 7-8, betreffend den Greek national action plan on asylum and migration.

59 Die drei Spruchkörper im „Europäischen Verfassungsgerichtsverbund“ haben mittlerweile festgestellt, dass ein Asylbewerber nicht an einen Mitgliedstaat - in den entschiedenen Fällen Griechenland - überstellt werden darf, in dem er Gefahr läuft, unmenschlich behandelt zu werden, s. EGMR, Urt. v. 21.1.2011, No. 30696/09 - M.M.S./Belgien und Griechenland, abgedruckt in: Europäische GrundrechteZeitschrift 38 (2011), 243; EuGH, verb. Rs. C- 411/10 und 493/10, Urt. v. 21.12.2011 - N.S. und M.E.; das beim BVerfG anhängige Verfassungsbeschwerde-Verfahren 2 BvR 2015/09 wurde mit Beschluss des Zweiten Senats v. 25.1.2012 eingestellt, nachdem das Bundesministerium des Innern das Bundesamt angewiesen hatte, generell von Überstellungen Asylsuchender nach Griechenland abzusehen und die Schutzgesuche im nationalen Verfahren zu prüfen. 
Fiskalvertrags vor dem Hintergrund der Geschehnisse in der Wirtschafts- und Währungsunion seit dem Frühjahr 2009 gefragt werden, ob der neue Vertrag angewendet werden wird.

Trotz der Meinungsverschiedenheiten über die Existenz und die Bedeutung eines finanziellen Einstandsverbots (no bail-out), ${ }^{60}$ dürfte mittlerweile außer Streit stehen, dass zumindest die mittelbare Finanzierung prekärer EU-Schuldnerstaaten durch die Europäische Zentralbank gegen das Primärrecht verstößt. ${ }^{61}$ Auch das freiwillige Helfen zwischen den Mitgliedstaaten hat Anfang des Jahres 2012 einen finanziellen Umfang erreicht, der der ursprünglichen Idee der Wirtschafts- und Währungsunion - Preisstabilität, Konvergenz, finanzielle Eigenverantwortung, Nicht-Haftung für fremde Schuld, Defizit- und Schuldenkontrolle - widerspricht. Wenn aber eine Lage eingetreten ist, in der die Verträge ihre Normativität nicht behaupten konnten, könnte dann nicht der Fiskalvertrag ebenfalls durch zukünftige Finanzmarkt-, Konjunktur- oder Schuldenkrisen unterspült werden? Kann sich vor diesem Hintergrund Vertrauen in die Normativität eines neuen Vertrages bilden?

Der in diesem Zusammenhang rechtfertigende Hinweis auf die erfolgreich in das Primärrecht eingegliederten, vormals intergouvernementalen Sachbereiche, ist ein hinkender Vergleich. Denn anders als in den Fällen des Abbaus der Personengrenzkontrollen (Schengen) und der Vertiefung einer europäischen Sozialpolitik parallel zum Maastrichter Vertrag (Sozialprotokoll), ${ }^{62}$ behandelt der Fiskalvertrag eine bereits im supranationalen Recht ausführlich geregelte Thematik: die Wirtschafts- und Währungsunion. Der Fiskalvertrag wird mit seiner Faktizität das europäische Recht materiell ändern - eine spätere formelle Vertragsänderung, dann im Konsens aller Mitgliedstaaten, wird das nur noch nachvollziehen.

60 Vgl. unter Fn. 22.

61 S. Art. 123 Abs. 1 AEUV; dem Argument, dass die EZB Art. 123 AEUV nicht verletzte, weil dort nur der „unmittelbare Erwerb von Schuldtiteln“ untersagt sei, ist der 7. Erwägungsgrund der VO (EG) Nr. 3603/93 v. 13. 12. 1993 entgegen zu halten, wonach die Beachtung der Haushaltsdisziplin nicht durch den Erwerb auf dem Sekundärmarkt umgangen werden darf. In Anbetracht der Höhe der eingesetzten Finanzmittel und der bewusst geförderten Erwartung, dass emittierte Schuldtitel von der EZB selbst oder durch entsprechend mit EZB-Geld ausgestattete Banken aufgekauft werden, liegt zweifellos eine Umgehung vor; kritisch auch Ruffert, M.: The European Debt Crisis and European Union Law, in: Common Market Law Review 48 (2011), 1777-1806, hier 1787-1788. Zu Sekundärmarktkäufen des ESM kritisch Deutsche Bundesbank: Monatsberichte, 2/2011, 72-73 und 8/2011, 66-69, hier Fn. 5.

62 Protokoll über die Sozialpolitik, dem ein Abkommen zwischen den Mitgliedstaaten der Europäischen Gemeinschaft mit Ausnahme des Vereinigten Königreichs Großbritannien und Nordirland über die Sozialpolitik beigefügt ist, BGB1. 1992 II, 1313. Das Abkommen wurde, nach der Zustimmung Großbritanniens, durch den Vertrag von Amsterdam in den EG-Vertrag übernommen (Art. 117-121 EGV a.F.). 
Vielleicht ist aber die hier aufgeworfene Frage nach der Normativität des Vertragsrechts bereits falsch gestellt: Gerade die Wirtschafts- und Währungsunion zeigt, dass das Recht als Institution unter einem politischen Primat steht. Weder das nationale Verfassungsrecht noch das Unionsrecht waren in der Lage, den politischen Führungswillen entscheidend zu begrenzen, der hinter den fortgesetzten sanktionslosen Defizitverstößen, der faktischen Änderung des Stabilitäts- und Wachstumspaktes durch Deutschland und Frankreich im Jahr 2003, ${ }^{63}$ der Festlegung des Kreises der Euro-Mitgliedstaaten ${ }^{64}$ und der Errichtung des Rettungsfonds stand und steht. Eindeutig ist die Äußerung eines ehemaligen Bundeskanzlers, der auf den Hinweis, dass seine Regierung die Kriterien für den Stabilitätspakt in Europa mit aufgeweicht habe, antwortete: „Wer die Kriterien des Stabilitätspakts als quasi von Gott gegeben hinnimmt, kann in Krisenzeiten nicht mehr adäquat reagieren. Hier geht es um genuin politische Entscheidungen. ${ }^{665}$ Die Krise macht auch den Blick frei auf das Rechtsverständnis europäischer Eliten und den geografisch radizierten Dissens über das Maß an Rechtsbindung durch die europäischen Verträge. ${ }^{66}$

\section{Sprossen auf der Leiter europäischer Integration}

Eine Krise ist nicht nur politisches Motiv für Rechtsetzung. Sie kann auch eine Chance für die Politik sein, lang gehegte Ziele „,im Windschatten der Krise“ in Recht umzusetzen. Die besondere Bedeutung von Finanz- und Schuldenkrisen für Staat und Gesellschaft ist verhältnisgleich zu den Gestaltungspotentialen von Krisenrechtsetzung. ${ }^{67}$

Der Fiskalvertrag ist nicht auf die Akutprobleme, sondern auf die Ursachen der gegenwärtigen Krise bezogen. Mit dem Vertrag wird das „fiskalische Rah-

63 Für die Ereignisse und deren unionsrechtliche Würdigung s. EuGH, Rs. C-27/04, Slg. 2004, I-6649 Kommission/Rat; dazu Palm, U.: Der Bruch des Stabilitäts- und Wachstumspakts Europäische Zeitschrift für Wirtschaftsrecht 15 (2004), 71-75; Deutsche Bundesbank: Monatsbericht, 4/2005, 15-21.

64 Loth, W.: Kreativ, vor allem in Buchführung. Wie Athen in die Eurozone gelangte und Berlin seinen Widerstand aufgab, in: Frankfurter Allgemeine Sonntagszeitung v. 18.9.2011, Nr. 37, 11.

65 Interview mit Gerhard Schröder, in: Passauer Neueste Nachrichten v. 9.9.2011.

66 Auf die unterschiedlichen Rationalitätsvorstellungen, Wertesysteme und Verhaltensnormen South of Paris und North of Paris verweist Hesse, J. J.: Die europäische Verschuldungskrise: eine dreifach unerledigte Agenda, in: ZSE 9 (2011), 338-351, hier 346; kritisch zu einer Nord-Süd-Dichotomie De Grauwe, $P .:$ The Eurozone as a Morality Play, in: intereconomic 46 (2011), 230-231.

67 Näher zu diesem Gedanken Schorkopf, F.: Finanzkrisen als Herauforderung für internationale, europäische und nationale Rechtsetzung, in: Veröffentlichung der Vereinigung Deutscher Staatsrechtslehrer (VDStRL) 71 (2012), i.E. Der Krisenbegriff ist hier als analytischer Begriff verwendet, um das Besondere einer Lage zu erkennen, vgl. Vierhaus, R.: Zum Problem historischer Krisen, in: Faber/Meier (Hg.): Theorie der Geschichte, München, 1978, 313-329, hier 315. 
menwerk“ - ergänzend zu der umfangreichen EU-Sekundärgesetzgebung überarbeitet, um Krisen vorzubeugen. ${ }^{68}$ Gleichwohl wird aus Anlass des Fiskalvertrages deutlich, dass die gegenwärtige Krise der EU zu einer politischen und institutionellen Vertiefung der europäischen Integration führen wird. Inwieweit der Fiskalvertrag dafür ursächlich ist oder seine Entstehung seinerseits nur den Krisenkontext spiegelt, verbleibt einstweilen im Dunst der Geschehnisse.

Zunächst ist das Beiseitestehen Großbritanniens und der Tschechischen Republik, kerneuropäische Mitgliedstaaten mit einem selbstbewussten Eigensinn für politische Freiheit, möglicherweise ein Akt europäischer Desintegration. Dass sich diese Mitgliedstaaten mit ihrer Position auf dem entscheidenden Gipfel am 9. Dezember 2011 nicht durchsetzten, gibt der Mehrheit kaum integrationsmoralische Überlegenheit - keiner der widerstreitenden politischen Standpunkte ist von vornherein besser oder schlechter. Das Ausweichen der Mehrheit der Mitgliedstaaten auf die intergouvernementale Ebene hat jedoch das Vertrauen in den politischen Meinungskampf auf europäischer Ebene erschüttert. Denn die Mitgliedstaaten müssen damit rechnen, dass sich unter Umständen die institutionellen Spielregeln unmittelbar verändern. Dem durchaus gewichtigen Gegenargument, dass besonders Großbritannien sich bewusst von der Wirtschafts- und Währungsunion absondere ${ }^{69}$ und deshalb die Euro-Staaten nicht an der Rettung ihrer gemeinsamen Währung hindern könne, ist die bisherige Praxis entgegen zu halten. Der Europäische Finanzstabilisierungs-Mechanismus (ESFM) wurde von allen 27 Mitgliedstaaten beschlossen und auch der Annahme der weitreichenden Regelungen des six-pack standen skeptische Mitgliedstaaten nicht entgegen. ${ }^{70}$

Die Intergouvernementalität hat in der Krise bereits an anderer Stelle deutlich sichtbar zur Desintegration geführt. Die bilaterale Rettung Griechenlands setzte

68 Deutsche Bundesbank: Monatsbericht, 2/2012, 64-69, hier 64.

69 Großbritannien ist nicht gezwungen oder verpflichtet, ohne einen gesonderten Beschluss seiner Regierung und seines Parlaments den Euro einzuführen; die britische Regierung hat den Rat am 16.10.1996 und am 30.10.1997 zudem darüber unterrichtet, dass sie nicht beabsichtigt, an der dritten Stufe der Wirtschafts- und Währungsunion teilzunehmen, s. das dem EUV und dem AEUV beigefügte Protokoll (Nr. 15) über einige Bestimmungen betreffend das Vereinigte Königreich Großbritannien und Nordirland, AB1. 2008 Nr. C 115/284.

70 Verordnung (EU) Nr. 407/2010 des Rates v. 11.5.2011 zur Einführung eines europäischen Finanzstabilisierungsmechanismus, ABl. EU 2011 Nr. L 118/1; die Verordnung ist formal nicht befristet und unterliegt lediglich einer Überprüfung durch die Kommission. S. auch Art. 8 RL 2011/85/EG, wonach das Kapitel über die Numerischen Haushaltsregeln (Art. 5-7) nicht für Großbritannien gilt; sowie die weiteren Sonderregeln in Art. 17 und Anhang der VO (EG) Nr. 14567/97 i.d.F. der VO (EG) Nr. 1177/2011, vgl. auch den 4. und 12. Erwägungsgrund. 
die politischen Integrationsmechanismen außer Kraft, sodass statt der Kommission mit ihrem technokratischen, aber überstaatlichen Habitus nun etwa der Bundesfinanzminister gegenüber dem griechischen Parlament, der Regierung oder direkt von den hellenischen Unionsbürgern ein bestimmtes Verhalten einfordert. Ressentiments erstarken, die „Europa“ doch ausgeräumt zu haben schien. Es ist vermutlich eine Ironie der Integration, dass diejenigen, die das Entstehen einer Politischen Union unumkehrbar machen wollten und wollen, durch die bewusste Inkaufnahme unzureichender Institutionen auf lange Sicht der Friedensfunktion europäischer Einigung widersprachen. Ein moderner europäischer Staatenkongress gehorcht einer politischen Ökonomie, die die europäische Integration zumindest nicht fördert. ${ }^{71}$

Die Mehrheit der Mitgliedstaaten könnte sich durch den Abschluss des Fiskalvertrages einer Logik der Vertiefung verschrieben haben. Wenn der europäische Integrationsverband nicht mehr einheitlich als EU-27 für die Fortentwicklung seines institutionellen Rahmens steht, muss sich eine ankündigende Spaltung politisch lohnen. Die Vorzeichen für einen erneuten großen Sprung hin zu einer Europäischen Verfassung, hin zu einer Europäischen Politischen Union, sind deutlich wahrnehmbar. Das bereits zitierte deutsche Konzeptpapier skizziert die Stabilitätsunion und sieht diese als Vorstufe zu einer Politischen Union. ${ }^{72}$ Anfang März 2012 warb der deutsche Außenminister auf dem informellen Gipfel der EU-Außenminister für eine europäische Verfassung, über die Vertreter von neun Mitgliedstaaten diskutieren sollen. ${ }^{73}$ Der Präsident des Europäischen Rates beschrieb in seiner Humboldt-Rede die gegenwärtigen Ereignisse nicht als eine

71 Eine bemerkenswerte Fußnote der Krise ist der Gebrauch militärischer und medizinischer Sprachbilder wie etwa „Ansteckungsgefahr“, „,gesunde Staatsfinanzen“, „Schuldenschnitt“, „Bazooka“, „Dicke Berta“, ausführlicher zur Herkunft des Krisenbegriffs Koselleck, R.: Einige Fragen an die Begriffsgeschichte von ,Krise', in: ders., Begriffsgeschichten, 2006, $203 \mathrm{ff}$., hier 204-205; Ricoeur, P.: Ist die „Krise“ ein spezifisch modernes Problem?, in: Michalski (Hg.): Über die Krise, 1986, 38ff,, hier 50-51.

72 Vgl. unter Fn. 6. Das Papier endet mit dem Satz: „Die Debatte über den Weg hin zu einer Politischen Union muss beginnen, sobald die Weichen in Richtung Stabilitätsunion gestellt sind.“ Die Euro-Gruppe könne unter der Führung Deutschlands und Frankreichs zum Kern einer weiter entwickelten Europäischen Union werden, Wieland, J.: Die Zukunft Europa - Krise als Chance, in: Juristenzeitung 67 (2012), 213-219, hier 219.

73 Frankfurter Allgemeine Zeitung v. 12.3.2012, 3. Der Gedanke einer europäischen Föderation wird in der Krise u.a. vertreten vom ehemaligen Präsidenten der EZB Trichet, J.-C.: Rede anlässlich der Verleihung des Internationalen Karlspreises zu Aachen v. 2.6.2011, 9-10; ders.: Trois ans après la chute de Lehman Brothers: leçons et défis de la crise, Rede v. 5.9.2011, zugänglich unter http://www.ecb.int/press; vom klassischen Standpunkt der europäischen Bewegung Duff, A.: Federal Union Now, 2011 sowie das „Schäuble-Lamers-Papier“ v. 1.9.1994; mit Bezug zu seiner Humboldt-Rede aus dem Jahr 2000 vgl. Fischer, J.: Es geht um fast alles!, in: Süddeutsche Zeitung v. 21.6.2011. 
„Renationalisierung der europäischen Politik“, sondern als eine „Europäisierung des nationalen politischen Lebens. “74

In dem Augenblick, in dem die Mechanismen staatlicher Eigenverantwortung und damit die Idee politischer Selbstbestimmung in Teilen der Europäischen Union versagen, ist das Rezept die weitere Integrationsvertiefung zwischen allen Mitgliedstaaten. Als politisches Motiv hat die Krise die Kraft, den Ordnungsrahmen eines Gemeinwesens in Frage zu stellen. Die Europäische Union befindet sich in solch einer Transformationskrise: Sie lässt einen Europäischen Staatshaushaltsfonds entstehen, erzwingt einen dauerhaften substantiellen Finanzausgleich, fordert eine einheitliche Wirtschaftspolitik und drängt zu einer zentralen Finanzaufsicht. Finanz- und Schuldenkrise werden so zu Sprossen auf der Leiter der Integration. ${ }^{75}$

\section{Verantwortungsgemeinschaften und verfassungsändernde Mehrheiten}

\section{Zustimmung und Ratifikation}

Ist der Fiskalvertrag vom Standpunkt des deutschen Verfassungsrechts tragbar? Die Bundesregierung hat den Ratifikationsprozess mit dem Hinweis eingeleitet, dass der Vertrag eine Zwei-Drittel-Mehrheit im Deutschen Bundestag benötige. ${ }^{76}$ Diese Mehrheit signalisiert eine Verfassungsänderung, was wiederum danach fragen lässt, welche Vertragsinhalte ein das Grundgesetz änderndes Moment haben. Die Bundesregierung erklärt, dass eine Zwei-Drittel-Mehrheit in Bundestag und Bundesrat entsprechend Art. 23 Abs. 1 Satz 3 i.V.m. Art. 79 Abs. 2 GG erforderlich sei, weil die deutschen Regelungen über die Schuldenbremse völkerrechtlich bindend werden würden und dadurch nicht mehr ohne Weiteres geändert oder ergänzt werden könnten. ${ }^{77}$

74 Herman van Rompuy: Die Erkenntnis der Mitverantwortung: Europa in der Schuldenkrise, Rede v. 6.2.2012, EUCO 21/12, 6: „Wir erleben gegenwärtig nicht eine ,Renationalisierung der europäischen Politik', nein, es handelt sich vielmehr um die ,Europäisierung des nationalen politischen Lebens'. Diese Veränderung mit all ihren Grenzen und Risiken wird das europäische Projekt letztlich nur weiter stärken."

75 In Anlehnung an Wolf, J.: Sozialismus und kapitalistische Gesellschaftsordnung, Stuttgart, 1892, zit. nach Koselleck, R., Stichwort: „Krise“, in: Geschichtliche Grundbegriffe, Bd. 3, 1982, 617ff,a.a.O., hier 644. Zur ideellen Aufladung des Integrationsbegriffs Schorkopf, F.: Der Europäische Weg, Tübingen, 2010, 178-182; zu Konvergenz und Angleichung als Motiven der Integration Wirsching, A.: Der Preis der Freiheit, München, 2012, 406-409.

76 Bundesregierung: Mitteilung v. 7.3.2012, Ein Vertrag für mehr Stabilität in der Wirtschafts- und Währungsunion.

77 Bundesregierung: Gesetzentwurf, a.a.O., BR-Drs. 130/12, 6. 
Dass die Bundesregierung auf den Fiskalvertrag also das deutsche Europaverfassungsrecht anwenden will, ist sehr bemerkenswert, wie ein Seitenblick auf den ESM-Vertrag zeigt. Beim ESM-Vertrag handelt es sich um einen klassischen völkerrechtlichen Vertrag, auf den die Bundesregierung Art. 23 GG ausdrücklich nicht anwenden will. Sie führt über die Frage sogar einen Organstreit vor dem Bundesverfassungsgericht, den die Fraktion Bündnis '90/Die Grünen beantragt hat. Die Fraktion macht für den Deutschen Bundestag geltend, dass die Bundesregierung das Parlament nicht angemessen über das Dossier ESM unterrichtet habe. ${ }^{78}$ Die Bundesregierung vertritt die Ansicht, dass es sich nicht um eine „Angelegenheit der Europäischen Union“ im Sinne des Art. 23 Abs. 2 GG handele. Wer diesen Standpunkt hat, der müsste konsequent die Auffassung vertreten, dass auch der Fiskalvertrag diese Voraussetzung nicht erfüllt - vielleicht hat die Bundesregierung aber auch politische Motive, sich auf die Zwei-DrittelMehrheit festzulegen ${ }^{79}$ oder aber sie nimmt nur einen Urteilsspruch aus Karlsruhe vorweg.

Die bereits skizzierten Kernregelungen des Fiskalvertrages lassen keine Zweifel daran, dass es sich um einen Vertrag handelt, der die primärrechtlichen Normen zur Wirtschafts- und Währungsunion vertiefen und ergänzen soll. Die Formulierung „,in Angelegenheiten der Europäischen Union“ betont den weiten Sinnzusammenhang, in dem der Anwendungsbereich des Art. 23 GG steht, und bezieht jeden unionsbezogenen Sachverhalt ein. ${ }^{80}$ Der Europa-Artikel ist unmittelbar auf den Fiskalvertrag anzuwenden. Der Vertrag enthält, vom deutschen Standpunkt aus betrachtet, jedoch keine Regelung, die nicht bereits der geltenden Schuldenbremse oder den unionsrechtlichen Pflichten entspräche und insoweit von den deutschen Zustimmungsgesetzen zum Primärrecht nicht gedeckt wäre. Das

78 Das Verfahren ist unter dem Aktenzeichen 2 BvE 4/11 beim Zweiten Senat des BVerfG anhängig und wurde am 11.11.2011, zusammen mit dem Verfahren über den so genannten ,9er-Ausschuss“ (2 BvE 8/11), mündlich verhandelt.

79 Christoph Möllers verweist in einer Notiz auf verfassungsblog.de v. 5.3.2012 darauf, dass der politische Prozess den Prüfungsmaßstab für ein zu erwartendes Verfahren in Karlsruhe anheben würde, d.h. diesen auf Art. 79 Abs. 3 GG verengen wolle. Ein „Vorteil“ der Anwendung des Art. 23 GG bestünde darin, dass der verfassungsändernde Gesetzgeber darauf verzichten könnte, bei der Ratifikation anzugeben, welche Grundgesetzartikel durch den Fiskalvertrag geändert werden; nach Wortlaut und herrschender Auslegung des Art. 23 Abs. 1 Satz 3 GG ist das Zitiergebot aus Art. 79 Abs. 1 Satz 1 GG nicht anwendbar.

$80 \S 3$ des Gesetzes über die Zusammenarbeit von Bundesregierung und Deutschem Bundestag in Angelegenheiten der Europäischen Union (EUZBBG) v. 12.3.1993 i.d.F. v. 22.9.2009, enthält keine abschließende Aufzählung der Vorhaben in der EU (,insbesondere“) - der Fiskalvertrag sieht außerdem vor, dass binnen fünf Jahren nach dem Inkrafttreten ein Versuch unternommen wird, seinen Regelungsgehalt zu supranationalisieren (Art. $16 \mathrm{FV}$ ). 
Grundgesetz wird inhaltlich nicht geändert oder ergänzt, solche Akte werden auch nicht ermöglicht. ${ }^{81}$ Das tragende Argument einer Konstitutionalisierung der Schuldenbremse, die das Instrument dem verfassungsändernden Gesetzgeber entziehe, beruht auf einem Irrtum. Der Fiskalvertrag ist ein völkerrechtlicher Vertrag, der nach den allgemeinen Regeln des Völkervertragsrechts gekündigt werden kann. ${ }^{82}$ Es ist hinzuzufügen, dass er nach der Verfassungsrechtsprechung sogar zu kündigen ist, wenn die Wirtschafts- und Währungsunion das Stabilitätserfordernis nicht mehr erfüllt. ${ }^{83}$ Nach dem Grundsatz der Völkerrechtsfreundlichkeit müsste der verfassungsändernde Gesetzgeber seinen Änderungswillen also lediglich aufschieben, bis der Vertrag wirksam beendet wäre.

Problematisch ist in diesem Zusammenhang die Rolle der Kommission. Die Pflicht aus Art. 3 Abs. 2 FV, eine Schuldenbremse nebst Korrekturmechanismus möglichst in das nationale Verfassungsrecht einzufügen, ist auf der Grundlage gemeinsamer Grundsätze zu erfüllen. Die Kommission wird die Grundsätze vorschlagen und derzeit ist nicht bekannt, wie diese aussehen werden. Es könnte sein, dass auch Deutschland seine geltende Schuldenbremse, die eines der Vorbilder für den Fiskalvertrag darstellt, in Einzelheiten wird ändern müssen ${ }^{84}$ Sollten die Grundsätze zu dem Zeitpunkt, in dem Deutschland den Vertrag ratifiziert, noch nicht vorliegen, hätte die Kommission plein pouvoir für die normative Konkretisierung der gemeineuropäischen Schuldenbremse und den Korrekturmechanismus erhalten.

Eine solche Ratifikation „ins Blaue hinein“, noch dazu im Finanz- und Haushaltsrecht, wäre mit dem Grundgesetz unvereinbar, ${ }^{85}$ so dass die Ratifikation in

81 Eine Grundgesetz-Änderung kann nicht mit Sicherheit ausgeschlossen werden, weil die Kommission erst noch ihre gemeinsamen Grundsätze für nationale Schuldenbremsen vorlegen muss, s. Fn. 84 und 85.

82 S. Art. 56 Abs. 1 lit. b des Wiener Übereinkommens v. 23. 5. 1969 über das Recht der Verträge (WVRK), der die Kündigung oder den Rücktritt von einem Vertrag auch ohne Beendigungsklausel u.a. für den Fall vorsieht, dass sich ein Kündigungs- oder Rücktrittsrecht aus der Natur des Vertrags herleiten lässt; Art. 60 WVRK regelt die Beendigung oder Suspendierung eines Vertrags infolge Vertragsverletzung. Daneben besteht das völkergewohnheitsrechtliche Vertragsrecht fort. Der Fiskalvertrag enthält kein Kündigungsrecht, aber auch keine Aussage, dass er auf unbestimmte Zeit geschlossen sei.

83 BVerfGE 89, 155 (205) - Maastricht; 123, 267 (395 f.) - Lissabon.

84 Die Bundesregierung erklärt in der Denkschrift zum Fiskalvertrag, dass die Frage der Folgeänderung im deutschen Recht im Zuge der Umsetzung des Art. 3 FV zu klären sei, BR-Drs 130/12, a.a.O., 2012, 20.

85 Bundesverfassungsgericht: Urteil des Zweiten Senats v. 7.9.2011 - 2 BvR 987/10, 2 BvR 1485/10 und 2 BvR 1099/10, Ziff. 125 - Griechenland-Hilfe/Euro-Rettungsschirm, auszugsweise abgedruckt in: Europäische Zeitschrift für Wirtschaftsrecht 22 (2011), 920-927: „Vor diesem Hintergrund darf der Deutsche Bundestag seine Budgetverantwortung nicht durch unbestimmte haushaltspolitische Ermächtigungen auf andere Akteure übertragen.“; zur „Bestimmtheit“ näher Nettesheim, M.: „Euro-Rettung“ und Grundgesetz, in: Europarecht 46 (2011), 765-783, hier 773-774. 
Bezug auf Art. 3 Abs. 2 FV nur unter Vorbehalt erfolgen könnte. Der Fiskalvertrag selbst enthält aber bereits einen solchen Vorbehalt, indem er darauf verweist, dass der Korrekturmechanismus die Vorrechte nationaler Parlamente uneingeschränkt wahrt. ${ }^{86}$ Die gemeinsamen Vorschläge der Kommission müssen so verstanden werden, dass sie nicht wortidentisch, sondern funktionsgleich in das nationale Recht zu übernehmen sind. Für die Verfassungsmäßigkeit des Fiskalvertrages entscheidend wird seine gesetzliche Einbindung in die innerstaatliche Willensbildung sein. So ist nach der neueren Rechtsprechung des Bundesverfassungsgerichts ${ }^{87}$ absehbar, dass etwa das deutsche Stimmrecht im Rat an eine vorherige parlamentarische Richtungsentscheidung rückgebunden wird; auf diesem Weg würde auch die Abstimmungsregel des Art. 7 FV verfassungsrechtlich verantwortbar. ${ }^{88}$

Der Fiskalvertrag verweist für eine parlamentarische Beteiligung auf eine Konferenz der jeweils zuständigen Ausschüsse des Europäischen Parlaments und der nationalen Parlamente (Art. 13 FV). Eine solche Konferenz sei im Rahmen des Selbstorganisationsrechts einzurichten, um Haushaltspolitik und andere Vertragsgegenstände „zu diskutieren“ - Selbstbestimmung wird durch Mitsprache ersetzt.

\section{Einwirkungen auf Politik, Wirtschaft und Gesellschaft}

Der in Kraft getretene Fiskalvertrag wird möglicherweise bestehende Zweifel daran verkleinern, dass die Schuldenbremse im Grundgesetz nicht angewendet werden könnte. ${ }^{89}$ Solche Zweifel bestehen, weil die strukturellen Haushaltsdefi-

86 Art. 3 Abs. 2 Satz 3 FV; gleichwohl ist die Haushaltsautonomie nur ,innerhalb des vom Vertrag vorgegebenen Rahmens“ gewahrt, s. Bundesregierung: Denkschrift zum Fiskalvertrag, BR-Drs. 130/12 (op. cit., Fn. 1), 22.

87 BVerfGE 123, 267 (406 ff.) - Lissabon; Urt. des Zweiten Senats v. 28. 2. 2012 - 2 BvE 8/11, Ziff. 132 ff. - Haushaltskernausschuss sowie BVerfG, a.a.O., 2011. Den Ansatz aus Gründen der demokratischen Legitimation befürwortend Calliess, C.: Der Kampf um den Euro: Eine „Angelegenheit der Europäischen Union“ zwischen Regierung, Parlament und Volk, in: Neue Zeitschrift für Verwaltungsrecht 31 (2012), 1-7.

88 Der Gesetzentwurf der Bundesregierung (BR-Drs. 130/12, a.a.O., 2012) sieht allerdings keine besonderen Beteiligungsrechte des Deutschen Bundestages vor. Der Entwurf eines Gesetzes zur finanziellen Beteiligung am Europäischen Stabilitätsmechanismus (ESM-Finanzierungsgesetz - ESMFinG) enthält einen Platzhalter (§ 3) für noch zu konkretisierende parlamentarische Beteiligungsrechte.

89 Ausweichmöglichkeiten der Finanzpolitik erörtert Mayer, C.: Greift die neue Schuldenbremse?, in: Archiv des öffentlichen Rechts 136 (2011), 266-322, hier 306-321. Bemerkenswert in diesem Zusammenhang ist die europäische Statistikregel, nach der Kredite des ESM, anders als Darlehen im Rahmen der Griechenland-Hilfe und der Euro-Rettungsfonds, in den Staatskonten der Euro-Staaten aufgrund der 
zite einiger Länder erheblich sind und auch der Bundeshaushalt sich nur bei besonders guter Konjunktur in der Nähe des eingebremsten Defizits bewegt. ${ }^{90}$ Wer die Defizitgrenzen einhalten will, der muss die Abgaben spürbar erhöhen oder konfliktgeneigte Verteilungsentscheidungen treffen; bei einem Wirtschaftsabschwung wird vermutlich beides notwendig sein.

Die Schuldenbremsen im Bund und in den Ländern werden die deutsche Politik sichtbar verändern, die mit dem Fiskalvertrag noch stärker als bisher durch die überstaatliche Rechtslage bedingt ist. Dabei werden neue Verbindungslinien hervortreten, die es bislang nicht gab oder die nicht wahrgenommen wurden: $\mathrm{Da}$ zu den so genannten makroökonomischen Ungleichgewichten auch Leistungsbilanzüberschüsse zählen, ${ }^{91}$ ist Deutschland nicht nur politisch, sondern auch unionsrechtlich gut beraten, sich einem bilanziellen Gleichgewicht anzunähern. Die Höhe der Lohnstückkosten wird in diesem Zusammenhang häufig genannt, sie hängt jedoch maßgeblich von den Entscheidungen der Tarifparteien ab. Der Fiskalvertrag adressiert diesen Zusammenhang in einem Erwägungsgrund der Präambel, indem die Notwendigkeit betont wird, bei der Vertragsumsetzung die jeweilige nationale Rolle der Sozialpartner zu achten. ${ }^{92}$ Gleichwohl lässt sich überspitzt formulieren, dass der Fiskalvertrag für Euro-Staaten wie Deutschland im Grunde eine völkerrechtliche Pflicht zur Lohnerhöhung enthält und damit mittelbar in den gesellschaftlichen Prozess der Lohnfindung eingreift.

Schließlich kann der Fiskalvertrag auch als Antithese in einem dialektischen Verhältnis von staatlichen Transfergebern und -empfängern verstanden werden. Erhalten die Euro-Staaten mit Leistungsbilanzüberschüssen eine rechtlich abgesicherte Begrenzung nationaler Verschuldungsmöglichkeiten, wird von ihnen zugleich die solidarische Finanzierung der Defizite erwartet. Ob dieser Zusammenhang bereits die Einführung von Euro-Bonds vorbereitet, ${ }^{93}$ kann dahin ste-

völkerrechtlichen Konstruktion des ESM nicht als Anstieg der öffentlichen Verschuldung angerechnet werden, vgl. Europäische Zentralbank: Monatsbericht, 7/2011, 89-90.

90 S. näher dazu Stabilitätsrat: Zusammenfassende Übersicht über die Beschlüsse des Stabilitätsrates zur Haushaltsüberwachung gemäß $§ 3$ Stabilitätsratsgesetz, 4. Sitzung v. 1. 12. 2011; Kennziffern zur aktuellen Haushaltslage und zur Finanzplanung im Stabilitätsrat, Gliederungspunkt „Kreditfinanzierungsquote".

91 Vgl. VO (EG) Nr. 1174/2011 und VO (EG) Nr. 1176/2011; konkret dazu aus volkswirtschaftlicher Sicht Busch, B./Grömling, M./Matthes, J.: Ungleichgewichte in der Eurozone, Köln, 2011.

92 17. Erwägungsgrund der Präambel FV. Der Erwägungsgrund wurde mit der dritten Entwurffassung aufgenommen und in der vierten Fassung substantiell geändert.

93 Europäische Kommission: Grünbuch über die Durchführbarkeit der Einführung von Stabilitätsanleihen, KOM (2011) 818 v. 23.11.2011; über solche Anleihen wird politisch und juristisch weiter intensiv nachgedacht, vgl. Sachverständigenrat, a.a.O., 2012, 106-118; Mayer, F./Heidfeld, C.: Aspekte der Ein- 
hen, verstießen solche Anleihen doch gegen das Grundgesetz. Mit der Haftung für Willensentscheidungen anderer Staaten würden schwer kalkulierbare Folgewirkungen übernommen werden. ${ }^{94}$ Die Verfassungsorgane würden sicherlich nicht sehenden Auges eine überstaatliche Maßnahme aushandeln und beschließen, die gegen das Grundgesetz verstieße.

\section{VI. „Dieses Mal ist alles anders“ - Europas wegen}

Die Krise der Europäischen Union wird als exzeptionell dargestellt, sie wird als noch nie dagewesen empfunden. Vergleiche zum „Großen Crash“ von 1929 werden gezogen und öffentliche Stellen sind auffallend bemüht, gegen solche vergleichenden Seitenblicke zu argumentieren. ${ }^{95}$ Dieses Mal sei die Krise anders, die Märkte seien schneller, die Geldsummen größer, die Welt vernetzter und die Sachzwänge drängender. Der historisch informierte Blick auf öffentliche Finanzen und Währungen macht allerdings bewusst, dass es über die Jahrhunderte erstaunliche Parallelen zwischen Krisen gegeben hat. ${ }^{96}$

Was dann aber doch die europäische Staatsschuldenkrise von den meisten Finanzkrisen früherer Jahrhunderte unterscheidet, ist die demokratische Legitimation ihrer Ursachen. ${ }^{97}$ Die Mitgliedstaaten beschlossen und ratifizierten die Wirtschafts- und Währungsunion 1992 zusammen mit dem Unionsvertrag von Maastricht. Wir wissen heute, dass es sich um ein Regelwerk handelte, das mit einer Mentalität entworfen wurde, wie sie zuweilen bei der Bautätigkeit der öffentlichen Hand anzutreffen ist: Ein Bauwerk wird begonnen, dessen Kosten nachlässig kalkuliert sind, dessen Plan vielleicht noch nicht fertig ist, für das aber die Alltagsregel gilt, dass es spätestens ab fertig gestelltem Rohbau zu Ende

führung von Eurobonds, in: Neue Juristische Wochenschrift 65 (2012), 422-427; Müller-Franken. S.: Eurobonds und Grundgesetz, in: Juristenzeitung 67 (2012), 219-225.

94 BVerfG, a.a.O., 2011, Ziff. 105 und 128.

95 Vgl. Bundesministerium für Wirtschaft: Monatsbericht 6/2009, 8-15; Europäische Kommission: Economic Crisis in Europe, European Economy 7/2009, 14 ff.; Internationaler Währungsfonds: World Economic Outlook, 2009, 99 ff.; zu Handlungszwängen aus dem historischen Kontext des Jahres 1929 Plumpe, W.: Wirtschaftskrisen, München, 2010, 120; Galbraith, J. K.: Der Große Crash 1929, München, 2008 (englische Erstausgabe 1954).

96 Reinhart, C./Rogoff, K.: Dieses Mal ist alles anders. Acht Jahrhunderte Finanzkrisen, München, 2010, die These des Buches steckt in der Ironie des Titels - die Krisen sind an sich jedes Mal gleich.

97 Vgl. Ohler, C.: Die Bewältigung der Schuldenkrise, Forum Constitutionis Europae (FCE 9/11), Vortragsmanuskript v. 1.11.2011, 2; auf die tatsächlichen und integrationspolitischen Folgen, die nicht eigenverantwortete Reformprogramme in den betroffenen Mitgliedstaaten wie Griechenland und Portugal haben könnten, verweist Scharpf, F.: Monetary Union, Fiscal Crisis and the Preemption of Democracy, in: ZSE 9 (2011), 163-198, hier 193-196. 
gebaut wird. Heute wird zuweilen die Erkenntnis der damaligen politischen Führung zitiert, dass eine Währungsunion auf Dauer ohne eine echte Wirtschaftsunion unmöglich sei. ${ }^{98}$ Warum wurde „Maastricht“ dann beschlossen? War es ein einzigartiges Experiment ${ }^{99}$ oder sollte ein Integrationsautomatismus in Gang gesetzt werden, dessen Hintergrundwirken heute sichtbar ist?

Die parlamentarischen Demokratien in der Europäischen Union haben außerdem eine Politik der Verschuldung der öffentlichen Hand betrieben, ohne die bewusst begründeten Vertragspflichten für eine begrenztes Haushaltsdefizit und eine begrenzte Staatsschuld einzuhalten. ${ }^{100}$ Mit dem Fiskalvertrag versucht die Mehrheit der Mitgliedstaaten nun, einen institutionellen Rahmen zu errichten, der diesen Weg für die Zukunft verschließt. Der Vertrag entpolitisiert die öffentliche Kreditaufnahme und technokratisiert die politische Steuerung mit der Ressource Geld. Es überrascht deshalb nicht, dass gerade auf der linken Seite des politischen Spektrums der Fiskalvertrag besonders kritisch wahrgenommen wird. Wenn dieser Zusammenhang von parlamentarischer Demokratie und öffentlicher Verschuldung richtig ist, dann ist das politische Bemühen um die weitere Parlamentarisierung der Europäischen Union zweischneidig. Die Europäische Union bemüht sich nämlich um neue Finanzquellen und hat die Hand nach europäischen Steuern und autonomer Kreditaufnahme ausgestreckt. ${ }^{101}$ Die EU, die den Weg in die Verschuldung einschlüge, würde sich, wie ihre Mitgliedstaaten, selbst überfordern.

98 Etwa Jacques Delors: „Wir stehen am Abgrund“, Interview mit der Frankfurter Allgemeinen Sonntagszeitung, 25.9.2011, Nr. 38, 6. Zu dem Zusammenhang von Währungs- und Wirtschaftsunion einerseits und politischer Verantwortung andererseits wieder lesenswert BVerfGE 89, 155 (207) - Maastricht. Beck, H./Prinz, A.: The Trilemma of a Monetary Union, in: intereconomics 47 (2012), 39-43, argumentieren, dass eine Währungsunion nicht gleichzeitig feste Wechselkurse, unabhängige zentrale Geldpolitik und selbständige nationale Fiskalpolitik garantieren könne.

99 Louis, J.-V., a.a.O., 2010, 607-608: „[...] the EMU, a unique and original experiment.“

100 Die Stimmen, die die Leistungsbilanzüberschüsse insb. Deutschlands als ursächlich für die Krise ansehen, wie etwa Scharpf, F.: Die Eurokrise: Ursachen und Folgerungen, in: ZSE 9 (2011), 324-337, hier 329-330, lassen die „Wirtschaftsverfassung“ der EU außer acht. Das Konzept des Binnenmarktes ist auf den freien Güterverkehr auf der Grundlage von Wettbewerb ausgerichtet - erst der Vertrag von Lissabon nimmt daran semantische Änderungen vor, indem er das soziale Element ergänzend erwähnt, zu diesen Ungleichgewichten vgl. auch Deutsche Bundesbank: Monatsbericht, 7/2010, 17-40.

101 Für entsprechende politische Initiativen für eine EU-Steuer vgl. Europäische Kommission: Mitteilung Überprüfung des EU-Haushalts, KOM (2010) 700, insb. 29-32; aus Sicht des Europäischen Parlaments: Bericht von Alain Lamassoure, Über die Zukunft der Eigenmittel der Europäischen Union, P6_A(2007)0066; vgl. auch die Forderung des für Haushalt zuständigen Kommissars Janusz Lewandowski, Financial Times v. 9. 8. 2010 (online-Ausgabe). Zum Vorhaben europäischer Infrastrukturanleihen („Projektbonds“) s. den Verordnungsvorschlag der Europäischen Kommission: KOM (2011) 659 endgültig, der sich einstweilen im Gesetzgebungsverfahren befindet. 
Der unterschiedliche Status der Mitgliedstaaten als Sanierungsstaaten und Staaten mit Leistungsbilanzüberschüssen trägt schließlich auch eine Debatte über die gerechte Güterverteilung in die Union hinein. Es wird versucht, sie über den Hinweis auf Solidarität zu lösen. ${ }^{102}$ Ein Teil der Mitgliedstaaten und ihrer Unionsbürger müssen solidarisch mit anderen Teilen der Union sein. ${ }^{103} \mathrm{Im}$ europäischen Repräsentationsorgan, dem Parlament, sind diese Interessen jedoch durch seine degressiv proportionale Zusammensetzung verzerrt abgebildet. Neuerdings werden sogar „Opfer“ für die Union eingefordert, als Gegenleistung für sechs Jahrzehnte europäischen Friedens. ${ }^{104}$ Einstweilen kann dieses Opfer für die dauerhaft friedliche Existenz eines deutschen Staates in Europa in Geld erbracht werden.

Der Fiskalvertrag ergänzt den errichteten Transferrahmen, um die Ursachen für den hohen Kreditbedarf einzelner Mitgliedstaaten zu beseitigen. Denn die „immer wieder aufgestockten Rettungsfonds können dagegen nur eins: Zeit kaufen. Zeit, die genutzt werden muss, um die grundlegenden Probleme zu lösen.“105

Wir können dem Pakt aus pragmatischen Gründen deshalb zustimmen, als Grundlage für ein werdendes Imperium müssen wir ihn ablehnen.

102 S. Böckenförde, E.-W.: Die Bedingungen europäischer Solidarität, in: ders., Wissenschaft, Politik, Verfassungsgericht, 2011, 267-280; ausführlich Calliess, C., a.a.O., 2011, 216-230; zur menschenrechtlichen Rückbindung Seibert-Fohr, A.: The International Covenant on Civil and Political Rights: Moving from Coexistence to Cooperation and Solidarity, in: Hestermeyer u.a. (Hg.): Coexistence, Cooperation and Solidarity, Bd. 1, Leiden, 2012, 521-552.

103 Skeptisch vor dem Hintergrund des Zusammenhanges von Finanztransfers und Zusammengehörigkeitsgefühl Di Fabio, U.: Europa in der Krise, in: ZSE 9 (2011), 459-464.

104 Hänsch, K.: Europas Sinn und Wert, in: Frankfurter Allgemeine Zeitung v. 27.2.2012, Nr. 49, 7. Der Beitrag endet mit dem Satz: „Die Anstrengungen und Opfer einer solidarischen Bewältigung der Krise werden die Zukunftsfähigkeit Europas neu beglaubigen. “

105 Weidmann, J.: 10 Jahre Euro-Bargeld, Rede v. 14.12.2011, Berlin, 8. 